\title{
1SXPS: A DEEP SWIFT X-RAY TELESCOPE POINT SOURCE CATALOG WITH LIGHT CURVES AND SPECTRA
}

\author{
P. A. Evans ${ }^{1}$, J. P. Osborne ${ }^{1}$, A. P. Beardmore ${ }^{1}$, K. L. Page ${ }^{1}$, R. Willingale ${ }^{1}$, C. J. Mountford ${ }^{1}$, \\ C. Pagani ${ }^{1}$, D. N. Burrows ${ }^{2}$, J. A. Kennea ${ }^{2}$, M. Perri $^{3,4}$, G. Tagliaferri ${ }^{5}$, and N. Gehrels ${ }^{6}$ \\ ${ }^{1}$ Department of Physics and Astronomy, University of Leicester, X-ray and Observational Astronomy Group, \\ University Road, Leicester, LE1 7RH, UK; pae9@leicester.ac.uk \\ ${ }^{2}$ Department of Astronomy and Astrophysics, Pennsylvania State University, University Park, Pennsylvania, PA 16802, USA \\ ${ }^{3}$ ASI-Science Data Center, Via del Politecnico, I-00133 Rome, Italy \\ ${ }^{4}$ INAF-Osservatorio Astronomico di Roma, Via Frascati 33, I-000040 Monteporzio Catone, Italy \\ ${ }^{5}$ INAF-Osservatorio Astronomico di Brera, via E. Bianchi 46, I-23807 Merate (LC), Italy \\ ${ }^{6}$ NASA/Goddard Space Flight Center, Greenbelt, MD 20771, USA \\ Received 2013 August 29; accepted 2013 November 20; published 2013 December 12
}

\begin{abstract}
We present the 1SXPS (Swift-XRT point source) catalog of 151,524 X-ray point sources detected by the Swift-XRT in $8 \mathrm{yr}$ of operation. The catalog covers $1905 \mathrm{deg}^{2}$ distributed approximately uniformly on the sky. We analyze the data in two ways. First we consider all observations individually, for which we have a typical sensitivity of $\sim 3 \times 10^{-13} \mathrm{erg} \mathrm{cm}^{-2} \mathrm{~s}^{-1}(0.3-10 \mathrm{keV})$. Then we co-add all data covering the same location on the sky: these images have a typical sensitivity of $\sim 9 \times 10^{-14} \mathrm{erg} \mathrm{cm}^{-2} \mathrm{~s}^{-1}(0.3-10 \mathrm{keV})$. Our sky coverage is nearly 2.5 times that of 3XMM-DR4, although the catalog is a factor of $\sim 1.5$ less sensitive. The median position error is $5^{\prime \prime} .5(90 \%$ confidence), including systematics. Our source detection method improves on that used in previous X-ray Telescope (XRT) catalogs and we report $>68,000$ new X-ray sources. The goals and observing strategy of the Swift satellite allow us to probe source variability on multiple timescales, and we find $\sim 30,000$ variable objects in our catalog. For every source we give positions, fluxes, time series (in four energy bands and two hardness ratios), estimates of the spectral properties, spectra and spectral fits for the brightest sources, and variability probabilities in multiple energy bands and timescales.
\end{abstract}

Key words: catalogs - methods: data analysis - surveys - X-rays: general

Online-only material: color figures

\section{INTRODUCTION}

Serendipitous X-ray source catalogs have been produced for most X-ray satellites since the Einstein mission (e.g., Gioia et al. 1990; Voges et al. 1999; Ueda et al. 2005; Watson et al. 2009; Evans et al. 2010) and have contributed much to our understanding of the X-ray sky. The Swift satellite (Gehrels et al. 2004) has several unique features which mean that a serendipitous source catalog produced from its X-ray Telescope (XRT; Burrows et al. 2005) can make a distinctive contribution to this field, particularly in the area of source variability. To make this catalog we have analyzed Swift-XRT data from the first 8 yr of operations, covering 13,065 distinct locations (giving a coverage of $1905 \mathrm{deg}^{2}$ ), of which $81 \%$ were observed at least twice. In many cases a field is observed both multiple times within a day and over a period of many days, allowing us to probe variability on different timescales. Swift pointings have been performed across the entire sky with considerable uniformity, although there is an overdensity of pointings along the Galactic plane; see Figure 1.

The XRT contains a CCD detector with a bandpass of $0.3-10 \mathrm{keV}$, with a peak effective area of $110 \mathrm{~cm}^{2}$ at $1.5 \mathrm{keV}$. The field of view has a radius of $12 ! 3$, with vignetting at the outer edge reducing the effective area by $\sim 25 \%$ (at $1.5 \mathrm{keV}$ ); there are also several detector columns permanently masked out due to damage from a micrometeoroid impact of 2005 May 27 (Abbey et al. 2006).

Two previous XRT point source catalogs have been produced, which used the routines built into the XIMAGE software to detect sources. The first, Puccetti et al. (2011), analyzed the deepest gamma-ray burst (GRB) fields, combining all of the data into a single image per field. The second, D'Elia et al. (2013), analyzed 7 yr of XRT data, considering each observation independently. For this catalog we have developed a new detection method capable of detecting fainter sources than these papers, and have conducted a rigorous analysis of our completeness and false positive rate; we have also considered both individual observations and deep images, making this a more complete point source catalog than those of Puccetti et al. (2011) and D'Elia et al. (2013). We have produced light curves and variability estimates for every source detected in the catalog. These are available through a dedicated Web site.

We performed our analysis in four energy bands: one covering the entire calibrated energy range of the XRT (0.3-10 keV), and three partial bands which were chosen to overlap those used in the 2XMM catalog (Watson et al. 2009); these are listed in Table 1. For a typical active galactic nucleus (AGN) spectrum this will give approximately the same number of events in each of the three partial bands. Summary details of the catalog are given in Table 1.

This paper is organized as follows. In Section 2 we discuss the data selection and filtering applied before collating the catalog. In Section 3 we detail the analysis process, the results of which are given in Section 4. In Section 5 we demonstrate the reliability of our catalog compilation, while Section 6 discusses the false positive rate and completeness.

\subsection{Data Timescales: Snapshots, Observations, and Stacked Images}

Swift data are organized into snapshots and observations. Due to its low Earth orbit ( $P=96$ minutes), Swift cannot observe an 


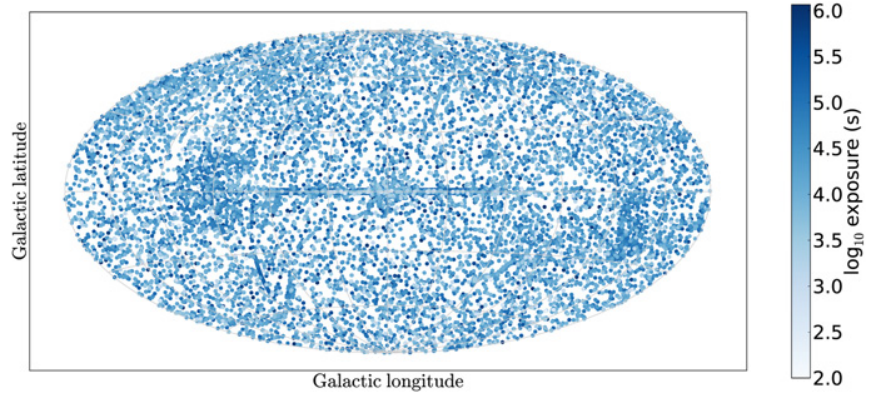

Figure 1. Locations of the observations in the 1SXPS catalog in Galactic coordinates. The colors of the points indicate the exposure time included in the catalog. The point sizes are larger than the XRT field of view.

(A color version of this figure is available in the online journal.)

Table 1

Summary Details of the Catalog

\begin{tabular}{lcl}
\hline \hline Category & Units & \multicolumn{1}{c}{ Value } \\
\hline Energy bands & $\mathrm{keV}$ & Total $=0.3 \leqslant E \leqslant 10$ \\
& & Soft $=0.3 \leqslant E<1$ \\
& & Medium $=1 \leqslant E<2$ \\
& & Hard $2 \leqslant E<10$ \\
Sky coverage & $\mathrm{deg}^{2}$ & 1905 \\
Median sensitivity $(0.3-10 \mathrm{keV})$ & $\mathrm{erg} \mathrm{cm}^{-2} \mathrm{~s}^{-1}$ & $3 \times 10^{-13}$ \\
Number of detections & & 585,443 \\
Number of unique sources & & 151,524 \\
Number of variable sources & & 28,906 \\
Number of uncataloged sources & & 68,638 \\
\hline
\end{tabular}

Note. ${ }^{a}$ That is, without a match within $3 \sigma$ in any of the catalogs detailed in Section 4.3 excluding the 2MASS and USNO-B1 catalogs.

object continuously for more than $2.7 \mathrm{ks}$, thus most observations are spread over multiple spacecraft orbits. A single, continuous on-target exposure is referred to as a snapshot. Within a UT day, ${ }^{7}$ the data from all snapshots pointed at a given source are aggregated into a single dataset, referred to as an observation and is assigned a unique ObsID under which the data can be accessed. In order to probe source variability we consider both of these timescales. Neither snapshots nor observations have a standard duration: snapshots may be $300-2700 \mathrm{~s}$ in duration ${ }^{8}$ and there are typically 1-15 snapshots in an observation. However snapshot-to-snapshot variability probes timescales $<1$ day, while observation to observation variability probes timescales $>1$ day.

Snapshots are generally too short for any but the brightest sources to be detected, therefore we search for sources in each observation and on summed images comprising all XRT observations on each location of the sky. We refer to these latter as stacked images. The word image where it appears in this paper can be taken literally as a single (FITS) image, which may be of a snapshot, observation or a stacked image; whereas field refers to an area on the sky. Figure 2 shows the distribution of exposure times in the two types of image on which we perform source detection, and the sky coverage of the catalog as a function of exposure time.

\footnotetext{
7 That is, from 00:00:00 to 23:59:59 UT.

8 Shorter snapshots are possible if a gamma-ray burst interrupts the planned observations.
}

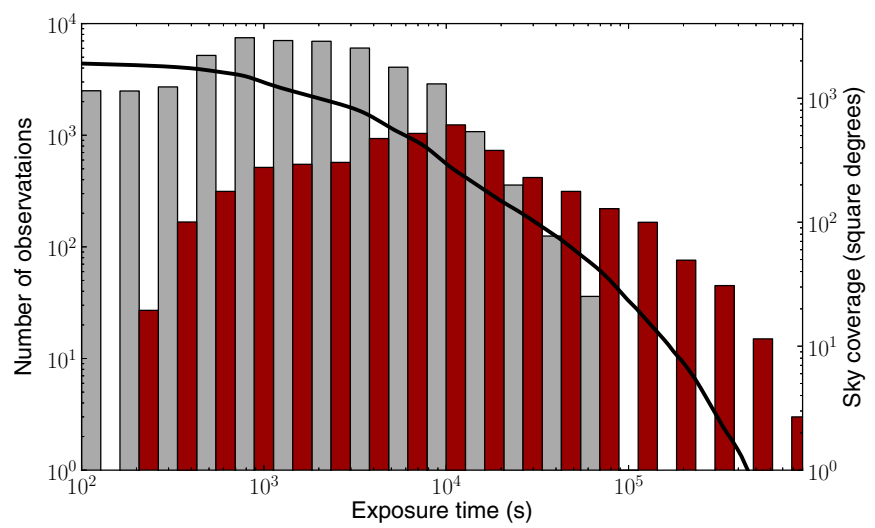

Figure 2. Temporal and geometric coverage of the 1SXPS catalog. The solid line shows the unique sky coverage of the catalog as a function of exposure time. The histogram shows the distribution of exposure times of the observations (gray) and the stacked images (red; darker gray in the printed journal).

(A color version of this figure is available in the online journal.)

\section{DATA SELECTION}

Initially we selected every XRT science observation ${ }^{9}$ collected before 2012 October 12 containing at least $100 \mathrm{~s}$ of photon counting (PC) mode data ${ }^{10}$; we also required that at least one snapshot in the observation was at least $100 \mathrm{~s}$ in duration. We removed any observations which overlap the locations listed in Table 2, as these include large-scale diffuse emission (identified by examining the XRT images) which is not well handled by our point-source-optimized detection system. We then filtered the remaining event lists to remove time intervals where the data were affected by light reflected off the sunlit Earth, or where the astrometry was unreliable (both described below); if this reduced the exposure time to below the $100 \mathrm{~s}$ limit, the observation was discarded.

\subsection{Bright Earth Filtering}

When Swift points close to the Earth limb, at certain spacecraft roll angles the background level in the XRT is increased by contamination from light scattered off the sunlit side of the Earth. This is always most notable on the left-hand side of the detector. For each observation we therefore examined the raw event list (before the XRTPIPELINE script has been executed) and selected events in a box $122 \times 350$ pixels in size, centered on the XRT detector pixel $(62,300)$ (i.e., the left-hand side). Times where the event rate in this box exceeds 40 event $\mathrm{s}^{-1}$ were deemed to be affected by bright Earth, and were removed from the observation before further processing. For $90 \%$ of the observations in our catalog, this removed less than $10 \%$ of the exposure time.

\subsection{Astrometry Filtering}

The standard astrometric calibration of XRT data is taken from the Swift star trackers, mounted on the XRT. This provides a solution which is accurate to 3.5 $90 \%$ of the time (Moretti et al. 2007). We identified and removed times where this astrometry was incorrect by more than $10^{\prime \prime}$ by using the UV/ Optical telescope (UVOT) on Swift. For each UVOT image we corrected the astrometry by matching UVOT sources to the

\footnotetext{
9 Excluding ObsIDs beginning with "006," as these are calibration datasets, sometimes taken in non-standard operating modes.

10 Windowed timing mode data have only one-dimensional spatial resolution so are inappropriate for detecting and localizing sources.
} 
Table 2

Locations Excluded from the Catalog Due to Large-scale Emission Structures

\begin{tabular}{|c|c|c|}
\hline $\begin{array}{l}\text { R.A. } \\
\text { (deg, J2000) }\end{array}$ & $\begin{array}{c}\text { Decl. } \\
(\mathrm{deg}, \mathrm{J} 2000)\end{array}$ & Identity \\
\hline 6.334 & 64.136 & Tycho SNR \\
\hline 16.006 & -72.032 & SNR B0102-72.3 \\
\hline 28.197 & 36.153 & RSCG15 \\
\hline 44.737 & 13.582 & ACO 401 \\
\hline 49.951 & 41.512 & NGC 1275 \\
\hline 81.510 & 42.942 & Swift J0525.8+4256 \\
\hline 83.633 & 22.014 & Crab nebula \\
\hline 83.867 & -69.270 & SN 1987A \\
\hline 85.052 & -69.331 & PSR 0540-69 \\
\hline 94.277 & 22.535 & OFGL J0617.4+2234 \\
\hline 116.882 & -19.303 & PKS 0745-191 \\
\hline 125.851 & -42.781 & Pup A \\
\hline 139.527 & -12.100 & Hydra A \\
\hline 161.017 & -59.746 & Carina nebula \\
\hline 177.801 & -62.626 & ESO 130-SNR001 \\
\hline 187.709 & 12.387 & M87 \\
\hline 194.939 & 27.943 & Coma cluster \\
\hline 207.218 & 26.590 & A1795 \\
\hline 227.734 & 5.744 & A2029 \\
\hline 229.184 & 7.020 & A2052 \\
\hline 234.798 & -62.467 & Swift J1539.2-6227 \\
\hline 239.429 & 35.507 & A2141 \\
\hline 244.405 & -51.041 & SNR G332.4-00.4 \\
\hline 258.116 & -23.367 & Ophiuchi cluster \\
\hline 266.414 & -29.012 & Galactic center \\
\hline 299.868 & 40.734 & $3 \mathrm{C} 405.0$ \\
\hline 326.170 & 38.321 & Cyg X-2 \\
\hline 345.285 & 58.877 & $1 \mathrm{E} 2259+586$ \\
\hline 350.850 & 58.815 & Cas A \\
\hline
\end{tabular}

Note. Observations within 12.5 of these locations are excluded from our catalog.

USNO-B1 catalog. We then determined the magnitude of this correction on the X-ray sources in the image and at four locations positioned symmetrically in the field at radii of 5.9 from the field center (i.e., mid-way to the edge of the field). This was done using the known translation from the UVOT detector to the XRT detector, as described in Goad et al. (2007). If any of these corrections were $>10^{\prime \prime}$ we marked the times of that UVOT image as bad and excluded XRT data taken during those times from the analysis. This was implemented as a two-pass process, since it makes use of the XRT source list for a given observation, which was not produced until the entire detection system had completed. We therefore ran the detect procedure on the per-observation timescale in full without this phase before performing this astrometric check. Any observations identified by this process were then reanalyzed from scratch, with the times of poor astrometry removed. The stacked images were only created and processed after this had been completed.

\section{DATA PROCESSING}

For all analyses in this catalog we used the HEASOFT version 6.12 software which includes the XRTDAS v2.8.0 developed at the ASI Science Data Center (ASDC, Italy), and the XRT CALDB version 20120209. Event files were reprocessed using the XRTPIPELINE task with the standard filtering criteria to provide a self-consistent and up-to-date set of event lists.

\subsection{Stacked Image Creation}

Our source detection software works in the SKY $(x, y)$ coordinate system, which is a virtual system constructed using a tangent plane projection such that $(x, y)$ has a linear mapping to (R.A., decl.) (see Greisen \& Calabretta 2002; Calabretta \& Greisen 2002). This coordinate system is produced uniquely for each ObsID when XRTPIPELINE is run. For the stacked images we therefore used the COORDINATOR FTOOL to reconstruct the coordinates for all observations within a stacked image using the same projection.

There is a small number of locations on the sky ( $4 \%$ of those covered by our catalog) where overlapping observations exist that extend beyond the $1000 \times 1000$ pixel $\left(=39^{\prime} .9 \times 39^{\prime} .3\right)$ range of the SKY coordinates in the XRT event files and thus could not be covered by a single stacked image. In these cases we split the observations into multiple stacked images, aiming to minimize the sky area lost while maximizing depth of exposure.

For stacked images of GRB fields we excluded the first snapshot of data from the stacked image as the GRB tends to be bright at this time which would reduce the sensitivity to fainter sources in the image.

From this point onward the process followed was the same for stacked images and single observations, ${ }^{11}$ and the phrase "dataset" refers to either of these.

\subsection{Data Preparation}

Source detection was performed on a single image (in each band) which contained all of the usable (Section 2) exposure time in that dataset. However, the background maps had to be created on a per-snapshot basis and then combined to give the full map (see Section 3.3.2 for details). The datasets were therefore split into snapshots, and for each snapshot an exposure map was created (which included the effects of vignetting, assuming an event energy of $1.5 \mathrm{keV}$ which is where the XRT effective area is at its highest) and an image was constructed of the grade 0-12 events in each of the four energy bands (Table 1). The center of the image and the mean spacecraft roll angle for that snapshot were recorded to be used by the background-mapping software. The XRT has three different window sizes that have been used at different times: $480 \times 480$ pixels, $500 \times 500$ pixels, and $600 \times 600$ pixels; the size that was used was also recorded. Finally, the per-snapshot exposure maps were summed to give a single, total exposure map (as well as the per-snapshot maps) as were the images in each energy band. These files were then passed to the source detection software.

\subsection{Source Detection}

Source detection was performed independently for each energy band. We used a form of sliding-cell detection combined with a fit to the point-spread function (PSF) to identify, localize and characterize sources. Our approach is based on that employed for the 2XMM catalog (Watson et al. 2009), optimized for Swift-XRT data. The algorithm is composed of the following elements:

1. Sliding-cell detection with a locally estimated background.

2. Creation of a background map.

3. Sliding-cell detection using the background map.

4. Source characterization using a PSF fit.

5. Likelihood testing.

11 With the exception that, for stacked images the data preparation phase is carried out for multiple event lists, once per observation in the image. 


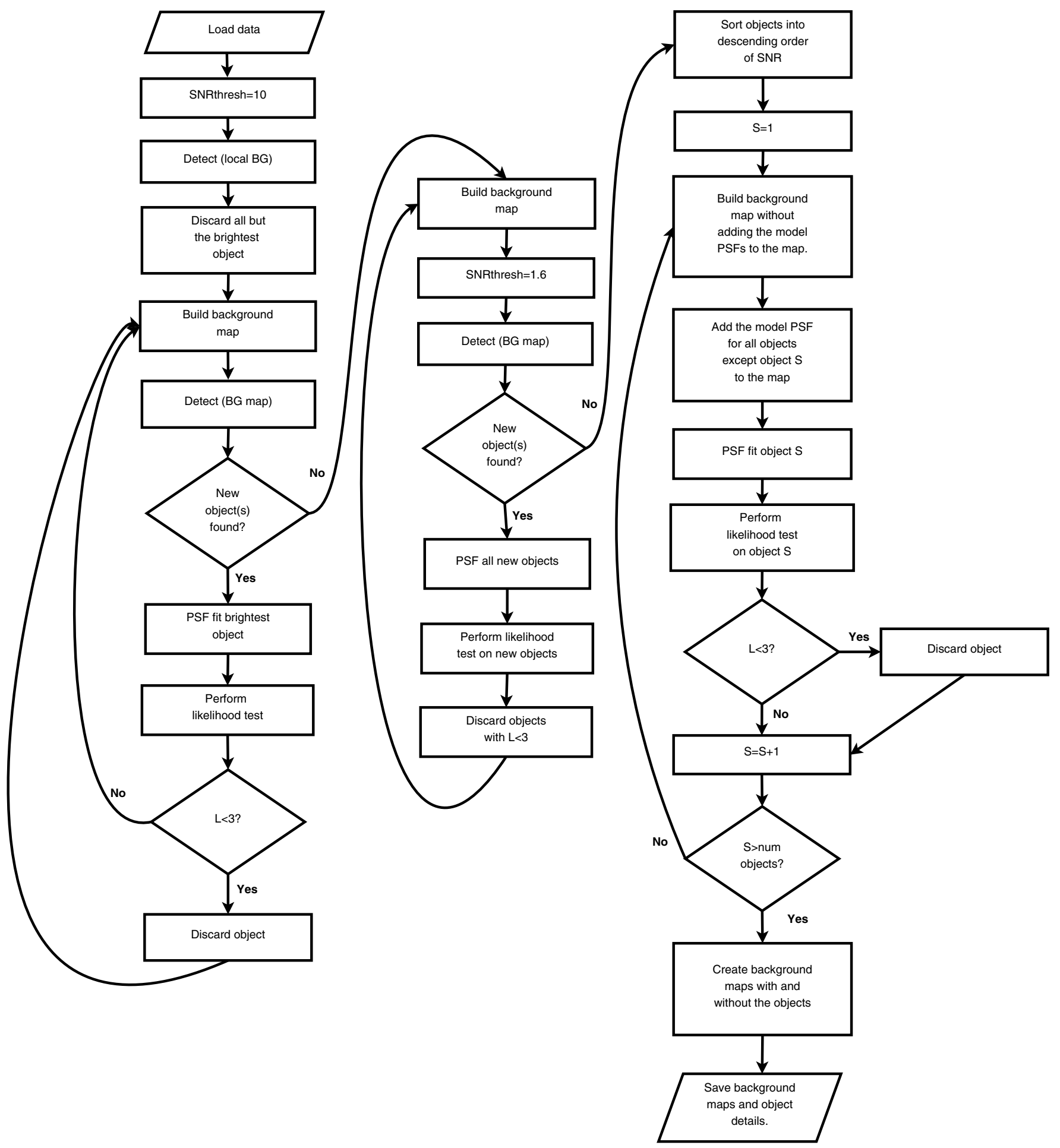

Figure 3. Flow chart showing the source detection and characterization algorithm.

The source detection process is nonlinear and iterative. The specific details (e.g., thresholds) and ordering of the steps were optimized through a series of trials and simulations. An overview of the algorithm is given in the rest of this section; the components of that algorithm are detailed in the following sections.

A flow-chart depicting the source detection algorithm is shown in Figure 3. The initial step was a sliding-cell detection with a locally estimated background. The source list thus produced was required only to produce the initial background map and only needs to identify the brightest sources. We therefore used a signal-to-noise $(\mathrm{S} / \mathrm{N})$ threshold of 10 . Only the brightest source was considered, and this was only used to create a background map, and then discarded. A second sliding-cell detection was then performed, this time using the background map. The $\mathrm{S} / \mathrm{N}$ threshold at this point was still 10, and only the single brightest source detected was kept. This is necessary to avoid detecting artifacts around bright sources. If a source was detected at this point, the PSF was fitted to the source and a likelihood test was performed. If the likelihood 
value (Section 3.3.5) was below 3, the source was discarded as spurious; its position was noted so that, if the object were redetected in a later step, it could be immediately discarded. The background map was then rebuilt, and the model PSF of the detected source was added to the map, which reduces the probability of detecting the artifacts just alluded to (see Section 3.3.2 for details). The siding-cell detection using the background map, and subsequent steps, were then repeated until no new objects were detected. These steps correspond to the lefthand column in Figure 3.

The detection threshold was then reduced to $\mathrm{S} / \mathrm{N}=1.6$ and the process continued largely as above (build background map, detect, PSF fit; repeat) except that all objects detected were passed to the PSF fit, rather than just the brightest one. This was repeated until no new objects were found. This stage is represented by the central column of Figure 3.

The final stage of the process was to perform a new PSF fit and likelihood test for each object detected. This was needed because the initial steps carried out above were done before all of the objects had been detected, so the background map will have evolved since this time. We therefore created the background map, adding in the model PSFs of all but the highest $\mathrm{S} / \mathrm{N}$ object. We then performed the PSF fit and likelihood test on the highest $\mathrm{S} / \mathrm{N}$ object, using this map. This process was then repeated with the second-highest $\mathrm{S} / \mathrm{N}$ object left out of the map (the highest $\mathrm{S} / \mathrm{N}$ source, relocalized in the previous iteration, is included) and the PSF fit and likelihood tests performed for that source, and so on through each source. Finally, two definitive background maps were created and saved: one containing only the background, one also including the model PSF of every object detected. These steps are shown in the right-hand column of Figure 3.

We will now describe the five principle components of this process.

\subsubsection{Sliding-cell Detection with a Locally Estimated Background}

Use of a locally estimated background was made only once in our process. During this phase the $\mathrm{S} / \mathrm{N}$ threshold for a detection was 10 . The algorithm employed was that detailed in the Chandra Detect Reference Manual. ${ }^{12}$ We used a $21 \times 21$ pixel $\left(=49^{\prime \prime} .5\right)$ cell and stepped it over the entire image in steps of 7 pixels. For each step, we measured the number of events, $C$ in the cell. The error was calculated according to the Gehrels (1986) formula:

$$
\sigma_{C}=1.0+\sqrt{C+0.75}
$$

which approximates the Poisson distribution better than $\sqrt{C}$ for low values of $C$.

We also measured the number of events, $T$ in a cell of size $51 \times 51$ pixels with the same central position as the source. If the real number of background events in the inner cell is $B$, and the number contributed by a source at the center of that cell is $S$, then:

$$
\begin{aligned}
& C=\alpha S+B \\
& T=\beta S+\left(\frac{b}{d}\right)^{2} B,
\end{aligned}
$$

where $\alpha=0.814$ and $\beta=0.937$ are the fraction of source counts expected in the inner and outer cell respectively, determined from the PSF of XRT Moretti et al. (2007); $d=21$ and $b=51$ pixels are the widths of the inner and outer cells.

\footnotetext{
12 http://asc.harvard.edu/ciao/download/doc/detect_manual/cell_theory.html
}

Solving for $B$ and then $S$ gives the $\mathrm{S} / \mathrm{N}$ in the inner cell:

$$
\mathrm{S} / \mathrm{N}=\frac{S}{\sigma_{S}}=\frac{C\left(b^{2}-d^{2}\right) d^{-2}-Q}{\sqrt{\sigma_{C}^{2}\left(b^{2}-d^{2}\right)^{2} d^{-4}+\sigma_{Q}^{2}}},
$$

where

$$
Q=T-C \text {. }
$$

This implicitly assumes that the exposure is constant across both the inner and outer cell, which may not be true. Therefore to determine $Q$, we measured the number of counts that were in the outer cell but not the inner cell, and then multiplied this by $E_{d} / E_{q}$; where $E_{d}$ is the mean exposure per pixel in the inner cell, and $E_{q}$ is the same calculated for pixels in the outer cell but not the inner one.

The 21 pixel wide cell is not necessarily optimal. We therefore searched for any 21 pixel cell with an $S / N \geqslant 1$, and then investigated such cells further, by creating a $17 \times 17$ pixel cell and stepping this around within the original 21 pixel cell (using an outer cell reduced in proportion). If one of these smaller cells had an S/N larger than was found in the 21 pixel cell, then its position and size were noted. The cell was then reduced to 15 pixels and stepped around inside the 21 pixel parent cell as before. This continued for cells of size 11,9 and 7 pixels, with the cell always being moved in steps of $d / 3$ pixels ( $d$ is the width of the cell, the step size is rounded when non-integer) until no cell with an $\mathrm{S} / \mathrm{N}$ greater than that in the 21 pixel region was found. Then all of the cells which were noted during this process were compared. If any cells overlapped, only that with the highest $\mathrm{S} / \mathrm{N}$ was kept. For each cell thus found, a barycenter was calculated (using only counts within that cell), and also the box size with the maximal $\mathrm{S} / \mathrm{N}$ was determined. If this box had $\mathrm{S} / \mathrm{N} \geqslant 10$ then it was saved as an "excess": a possible source.

Once the entire image had been searched in this way, any duplicate excesses were removed. If there were overlapping excesses, ${ }^{13}$ the mean box size and position is determined, weighted according to the number of events in each cell. A barycenter was then calculated, and the overlap check repeated; this time where excesses overlap, only that with the highest S/ $\mathrm{N}$ was kept; the others were discarded. The final result of this process was a unique list of excesses with $\mathrm{S} / \mathrm{N} \geqslant 10$.

\subsubsection{Creating a Background Map}

The above method assumes that there is at most a single source within the test cell; where multiple sources are close together this will therefore incorrectly estimate the background level. It also assumes that the cell is large enough to accurately sample the background and that this is invariant across the cell. These statements may be untrue.

We therefore produced background maps to accurately model the background across the detector and included in this map the sources which had already been detected. This process was repeated many times during the source detection process and it is pivotal to our method: in Section 6.1 we demonstrate that it is reliable.

Even within a single observation, each snapshot covers a slightly different area of sky because it follows a new slew to the target. If we created a background map based on the full exposure, this would contain artifacts at the edges of the persnapshot fields of view (particularly if the background level varies between snapshots, for example due to thermal variations

\footnotetext{
13 Because the 21 pixel cells are moved in steps of 7 pixels and thus overlap.
} 


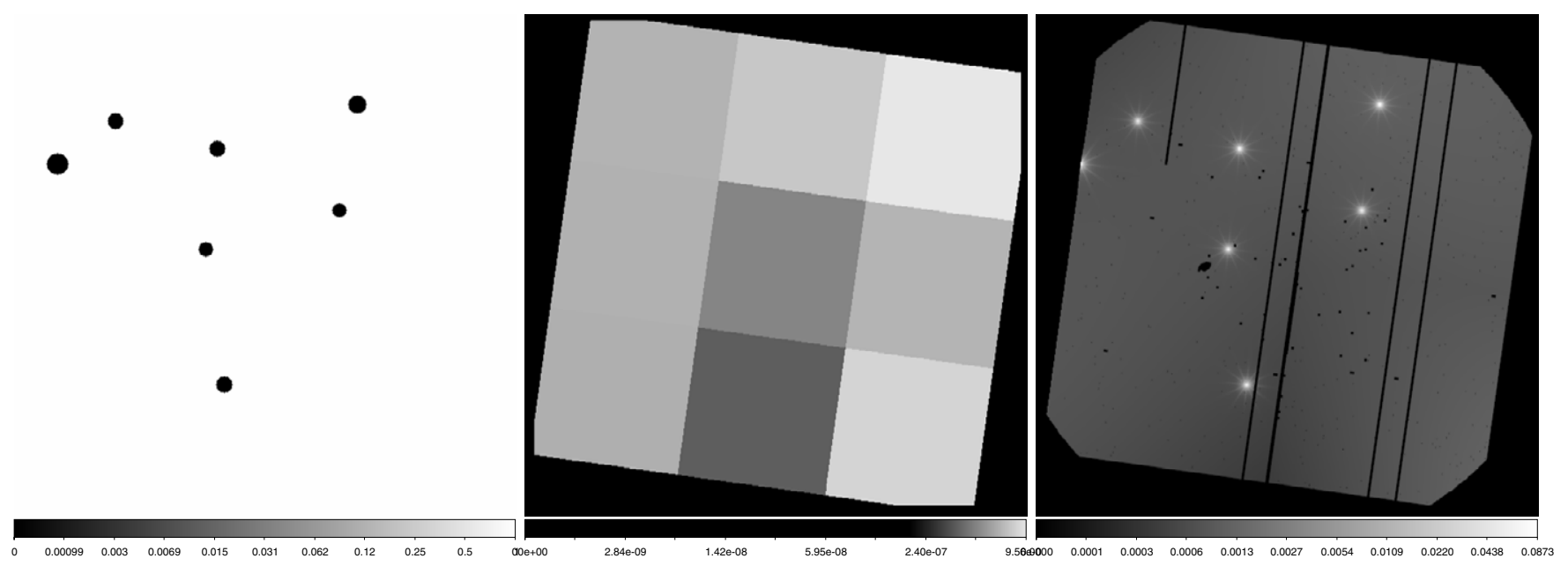

Figure 4. Example stages of background map creation on a single snapshot. Left: the detector mask; white pixels are "on" while black ones are masked out. Center: the rebinned background. Right: the final background map, including the model PSFs of the sources detected so far.

in the passively cooled XRT; Kennea et al. 2005). We therefore constructed the background map separately for each snapshot using the images and exposure maps created in Section 3.2, and then summed these to create the per-image background map. The process, described below, makes a single-snapshot, singleband background map, and was performed for each energy band and snapshot independently.

The first step was to create a detector mask. Initially all pixels in the mask were set to 1 , then all pixels in the region of the excesses already identified were set to 0 . The definition of "in the region of" depended on the details of the excess. For all but the first and last background maps created for a dataset, the list of excesses comprised a mixture of those returned by the most recent cell detect run and those which had been PSFfitted. For the former, the position and count rate were not well known, so the masking was approximate: the count rate of the excess was estimated based on the size of the cell in which the excess was detected and the standard XRT PSF, and pixels were masked out to the radius at which the count rate dropped below $10^{-5}$ count s$^{-1}$ pixel $^{-1}$ (or a maximum radius of 150 pixels). For PSF-fitted excesses the best-fitting PSF profile and count rate were known, so the mask radius was that where the count rate fell to $10^{-6}$ counts s ${ }^{-1}$ pixel $^{-1}$ (a typical background level for an XRT exposure), again with a maximum of 150 pixels. If this process resulted in more than $80 \%$ of the image being masked out, the mask radius was reduced by $5 \%$ and the process reperformed; this was repeated until less than $80 \%$ of the image was masked (we set a maximum of 100 iterations, but this was never reached).

The mask was multiplied by the original image to create a masked image (referred to as a "Swiss-cheese image" by Rosat and XMM; Voges et al. 1999; Watson et al. 2009), i.e., one where ideally all events from the detected sources have been removed. This image was divided by the exposure map ${ }^{14}$ and rebinned into a $3 \times 3$ grid, with the uncertainty in each bin also calculated according to Equation (1). If a box contained no unmasked pixels, the value of that box was set by interpolation from the neighboring boxes. The central pixel of each box was set to the value determined for that box, and the rest of the image was populated using bilinear interpolation from these nine values. The resultant image was then multiplied by

\footnotetext{
${ }^{14}$ Pixels with zero exposure are set to zero.
}

the exposure map to give the background map. This process differs from the $X M M$ approach of using spline interpolation over a finer grid than employed here, however that process tended to overfit the Swift background. The above approach of linear interpolation and a $3 \times 3$ grid was arrived at through an extensive period of testing, and represents an excellent level of accuracy (Section 6.1) for a modest number of parameters. The uncertainties were propagated through this process to give a background error map.

Once the background had been modeled in this way, any excesses which had been PSF-fitted in previous iterations were added to the background (and background error) map; to reduce the number of spurious detections near to bright sources, and increase sensitivity to sources which are close together. This was done using the PSF profile from the PSF fit (Section 3.3.4) which has been modified to include the spokes caused by the shadow of the mirror support structure, and out-of-time (OOT) events (see the Appendix).

The creation of a background map is illustrated in Figure 4.

\subsubsection{Sliding-cell Detection Using the Background Map}

This process was almost identical to that described in Section 3.3.1, except that the outer cell was not used. Instead the background level, $B$ was simply the sum of the background map within the cell, and hence

$$
\mathrm{S} / \mathrm{N}=\frac{S}{\sigma_{S}}=\left(\frac{C-B}{\sqrt{\sigma_{C}^{2}+\sigma_{B}^{2}}}\right),
$$

where $\sigma_{C}$ was defined as in Equation (1) and $\sigma_{B}$ was taken from the background error map.

\subsubsection{Source Characterization Using a PSF Fit}

The positions of the excesses were determined using a PSF fit based on that described in Goad et al. (2007) and Evans et al. (2009). A circular region was selected, centered on the position determined by the sliding-cell detection, with a radius based on the $\mathrm{S} / \mathrm{N}$ of the excess as given in Table 3 . The best position of the source was then determined by minimizing the $C$-stat (Cash 1979) as modified for use in XSPEC

$$
\mathcal{C}=2 \sum_{i}\left(M_{i}-D_{i}+D_{i}\left[\ln D_{i}-\ln M_{i}\right]\right)
$$


Table 3

The Radius of the Region Used to Perform PSF Fitting

\begin{tabular}{lc}
\hline \hline $\mathrm{S} / \mathrm{N}$ & Radius $^{\mathrm{a}}$ \\
\hline $\mathrm{S} / \mathrm{N} \leqslant 7$ & 12 pixels \\
$7<\mathrm{S} / \mathrm{N} \leqslant 11$ & 15 pixels \\
$11<\mathrm{S} / \mathrm{N} \leqslant 40$ & 20 pixels \\
$\mathrm{S} / \mathrm{N}>40$ & 30 pixels \\
\hline
\end{tabular}

Note. ${ }^{\text {a }} 1$ pixel $=2$.' 357 .

where the sum is over all pixels in the circular region, $D_{i}$ is the number of events measured in a pixel $i$, and $M_{i}$ is the expected number of events in that pixel:

$$
M_{i}=E_{i}\left(N P_{i}+B_{i}\right) \text {, }
$$

where $E_{i}$ is the exposure, $N$ is the normalization, $P_{i}$ is the model PSF and $B$ the value of the background map, in pixel $i$. We fitted for source position and normalization, using both the nominal PSF in the CALDB (Moretti et al. 2007) and the PSFs determined for piled-up sources (Evans et al. 2009); these were first modified to include the shadows of the telescope's mirror support structure (see the Appendix for details). Based on simulations, we required that $\mathcal{C}$ decrease by at least 10 before accepting a more piled-up PSF as a better fit. Although the PSF is a function of both energy and off-axis angle, the dependence on these factors is very small and we used the onaxis $1.5 \mathrm{keV}$ profile for all of our fits. The $68 \%$ confidence intervals on the R.A. and decl. were determined independently, by finding for each parameter the range of values within $\Delta \mathcal{C}=1$ of the best-fitting $\mathcal{C}$ value. This was later converted to a $90 \%$ confidence radial error via Rayleigh statistics, using $\sigma_{\text {Rayleigh }}=0.5 \times\left(\sigma_{x}+\sigma_{y}\right)$. For a small number of objects, the fit was unable to determine the uncertainty due to minimization errors. In these cases we set the $90 \%$ confidence radial error to be 14 .' $6 / \sqrt{N}$ (where $N$ is the number of events in the fitting region), this relationship having been calibrated from simulations.

We then reconstructed the count rate of the source, needed for the background map. For most sources this was done using a circular region with radius as for the PSF fit, but centered on the position returned by that fit. However, if the best-fitting PSF was one of the piled-up profiles, or if the estimated count rate in the original circle was $>0.6$ counts $\mathrm{s}^{-1}$ (the level at which pile-up tends to become significant) an annular region was instead used, with the inner radius given in Table 4; these reflect the radii at which the piled-up PSFs become asymptotic to the non-piled-up PSF. The outer radius was still that used for the PSF fit if this was larger than the inner radius, otherwise it was 5 pixels more than that value.

The measured and background counts, $C$ and $B$, were taken from the image and background map respectively in the region just defined. If $(C-B)>30$ then the estimated number of source events, $S=C-B \pm \sqrt{C+B}$ as in Equation (6) (except that we define $\sigma_{C}=\sqrt{C}$ as we are no longer in the low-count regime). For lower numbers of measured counts the value $S$ was determined using the Bayesian method of Kraft et al. (1991). ${ }^{15}$ To correct for the effects of pileup, vignetting and exposure

15 At $C-B=30$ the Bayesian calculation converges with the standard approach. However, the Bayesian approach assumes that there is no uncertainty in the background measurement which in principle leads to an underestimate of the error. For typical detections in our catalog this is at the $0.1 \%$ level, so can be ignored.
Table 4

The Inner Radius of the Annular Region Used to Measure the Count Rate for Piled Up Sources

\begin{tabular}{|c|c|}
\hline Fitted PSF Profile & Radius \\
\hline CALDB & 3 pixels \\
\hline Rate $=0.9$ counts $\mathrm{s}^{-1}$ & 4 pixels \\
\hline Rate $=1.4$ counts $\mathrm{s}^{-1}$ & 6 pixels \\
\hline Rate $=2.6$ counts $\mathrm{s}^{-1}$ & 7 pixels \\
\hline Rate $=4.0$ counts $\mathrm{s}^{-1}$ & 8 pixels \\
\hline Rate $=5.2$ counts $\mathrm{s}^{-1}$ & 13 pixels \\
\hline Rate $=8.6$ counts $\mathrm{s}^{-1}$ & 20 pixels \\
\hline Rate $=15$ counts $s^{-1}$ & 25 pixels \\
\hline
\end{tabular}

Notes. The "CALDB" profile is that determined by Moretti et al. (2007) and given in the CALDB. The remainder were determined by Evans et al. (2009). The "rate" is related to the object used to calibrate the PSF and not to the source being characterized in this catalog. The PSF profile used to determine the count-rate correction factor is the one determined in the PSF-fitting stage.

Table 5

The Distance from a Source within Which Detections are Assumed to be Artifacts

\begin{tabular}{lc}
\hline \hline $\begin{array}{l}\text { Source Rate } \\
\left(\text { count } \mathrm{s}^{-1}\right)\end{array}$ & $\begin{array}{c}\text { Radius } \\
\text { (pixels) }\end{array}$ \\
\hline$R \leqslant 0.4$ & 10 \\
$0.5<R \leqslant 1$ & 35 \\
$1<R \leqslant 2$ & 40 \\
$2<R \leqslant 8$ & 47 \\
$R>8$ & 70 \\
\hline
\end{tabular}

variations (e.g., due to dead columns on the CCD) we calculated the correction factor:

$$
\kappa=P_{\text {inf }} / P_{\text {meas }},
$$

where

$$
\begin{aligned}
P_{\text {inf }} & =\sum_{i}^{r=150}\left(E_{i m} P_{i}\right) \\
P_{\text {meas }} & =\sum_{i}\left(E_{i} P_{i}\right) .
\end{aligned}
$$

$P_{\text {inf }}$ is the PSF summed from a radius of 0 out to 150 pixels (effectively infinity), while $P_{\text {meas }}$ was summed only over the region from which counts were measured. $E_{i m}$ is the on-axis exposure of the image. The estimated source count rate is thus:

$$
R=\frac{\kappa S}{E_{i m}} .
$$

We next checked for potential duplicates or detections of the same astrophysical object. These can occur in the PSF wings and diffraction spikes of bright sources, even though these were added to the background map at each iteration. We therefore checked the distance of each newly fitted excess from those found in previous iterations. If it lay within the distance tabulated in Table 5 it was assumed to be an alias of that object, and was discarded. This means that our detection method is blind to new sources in the close vicinity of brighter objects, however the tendency to detect false positives in this region had effectively blinded the system anyway. Due to the nature of 
Swift's observing strategy, this limit is often only temporary. For example, a newly detected GRB is usually bright, so the radius over which we cannot detect new sources is large, however the GRB is observed again as it fades; in those later observations sources close to the GRB can be reliably detected.

\subsubsection{Detection Likelihood}

After PSF-fitting an excess we calculated $\mathcal{C}$ a second time with the normalization set to 0 , i.e., with no source present. Since $\Delta \mathcal{C}$ is distributed as $\Delta \chi^{2}$ (Cash 1979; here with 2 dof, $v=2$ ) we determined the probability that the change in fit statistic with and without a source present is coincidence: $P=\Gamma(v / 2, \Delta \mathcal{C} / 2)$ (where $\Gamma$ is the incomplete Gamma function), and the loglikelihood, $L=-\ln (P)$. As Watson et al. (2009) pointed out, we cannot take this statistic at face value; indeed the false positive levels they report are 10-100 times higher than expected from the equations above for the likelihood values they quote. This is because the measurement with no source present is a boundary condition of the model: as the source normalization cannot be negative, the test with normalization set to 0 is at the limit of the allowable model space. In such cases the likelihood ratio does not follow a $\chi^{2}$ distribution (see Protassov et al. (2002) for a detailed discussion). Like Watson et al. (2009) we instead calibrated the relationship between $L$ and $P_{\text {false }}$ using simulations, as described in the Section 6. Based on this calibration, we rejected any excess with $L<3$.

\subsection{Quality Flags and Further Checks}

Several further tests were performed to eliminate spurious or extended sources and to indicate how reliable a given detection is. Spurious detections can arise due to hot columns and hot rows on the detector. For each excess, we selected from the relevant event list all the events lying within the PSF-fitted region. Only excesses containing events from at least three distinct detector pixels, rows and columns were accepted; in addition, any excess where $>50 \%$ of the events lie in a single pixel, or $>75 \%$ lie within a single row or column was discarded. After this the location of each surviving excess was compared to a list of known extended objects (taken from Tundo et al. 2012): if the excess lay within the extent of the extended object it was discarded.

The remaining excesses are considered to be detections of genuine astrophysical sources, but some level of contamination will remain: we therefore assigned each source a quality flag to indicate the probability that it is a false positive. This flag is a function of the exposure time and the likelihood value for the source, and can be either Good, Reasonable or Poor (with corresponding integer values of 0,1 , and 2). If only Good sources are considered, the false positive rate is $0.3 \%$; if Good and Reasonable sources are included, this rises to $1 \%$, and if Poor sources are also considered, the false positive rate is $10 \%$. Of course the fraction of true sources that are detected (i.e., the completeness) also rises as Reasonable and Poor detections are included. This allows users to easily choose between sample size and sample purity. Full details of the definitions of the quality flags and how the false positive rate and completeness fraction were calibrated are given in Section 7.

There is an additional category of sources, Bad, which is not included in our catalog. Such sources were accepted by the source detection code, but as they have a very high false positive rate $(\sim 80 \%)$ they were rejected before the detections are merged (Section 3.5). The background map was reconstructed at this point without the Bad detections considered. This new background map was used for construction of the source count rates and light curves. We stored a list of these Bad detections for use with the upper limit server (Section 4.4).

We also performed an automated check for the phenomenon called optical loading. Bright optical sources can liberate sufficient charge in the XRT CCD because of the large number of optical photons accumulated in a $2.5 \mathrm{~s}$ PC mode exposure frame that the characteristics of X-ray events at the location of the optical source are distorted. When this first becomes a problem, it causes the energy of the X-ray events to be overestimated. ${ }^{16}$ At higher optical fluxes, it can cause real X-ray events to be discarded or spurious events to be detected. The flux at which this occurs is a function of stellar color and is discussed in detail at http://www.swift.ac.uk/analysis/xrt/optical_loading.php; stars brighter than $V \sim 9$ can be a problem, the limit being more severe for those later than $M 0$. We set a threshold at which optical loading is to be flagged as that at which a star contributes spurious events at a level of $\geqslant 10^{-3}$ counts $\mathrm{s}^{-1}$. We searched for cataloged stars above this threshold within $30^{\prime \prime}$ of each X-ray source in our catalog, using their cataloged $B-V$ color to estimate spectral type and hence determine the $V$ magnitude limit. If such a star was found, a field OL_WARN is set in the catalog, indicating how many magnitudes brighter than the threshold the star is. We used the Tycho-2 (Høg et al. 2000), Bright Star Catalog (Warren \& Hoffleit 1987) and General Catalog of Variable Stars (Samus et al. 2010) as our source of optical objects. These sometimes contain the peak magnitude of a variable object, which may not be appropriate to the Swift observations (e.g., GK Perseii has a catalog magnitude of $\sim 0$, based on its nova eruption of 1908; but was at least 10 mag fainter during all Swift observations), so a large OL_WARN value should be taken as a warning that an object may be affected by optical loading, rather than that it is affected.

\subsection{Merging Detections Across Bands}

Since the detection system was performed independently on the four energy bands within a dataset, the list of sources detected in each band had to be merged to create a unique list of sources for that dataset. This was done by considering the detected sources in descending order of $\mathrm{S} / \mathrm{N}$ and then using the radii given in Table 5 to determine which detections correspond to the same object. Where a source was detected in multiple energy bands, the definitive position of that source (in this dataset) was taken from the detection with the smallest position error (provided this is not one where the error could not be determined from the fit).

For a source which was undetected in one or more energy bands, the images of those bands were examined to determine the number of events at the source location. The expected background level was determined from the corresponding background map. The count rate and error for this energy band was then estimated using the Bayesian approach of Kraft et al. (1991) and the PSF correction $\kappa$ was applied as for detected sources. Although the source was undetected in this case, we did not produce an upper limit, even though the count rate may well be consistent with zero. Instead we determined the value and the $68 \%$ (i.e., $1 \sigma$ ) confidence limits, as we do for detections. Note that we give the positive and negative uncertainties separately as, when using the Bayesian approach, they may not be the same.

\footnotetext{
16 A correction for this is made by the XRTPCCORR tool called by XRTPIPELINE
} as part of the standard processing. 
We also determined two hardness ratios, defined as

$$
\begin{aligned}
& \mathrm{HR} 1=(M-S) /(M+S) \\
& \mathrm{HR} 2=(H-M) /(H+M),
\end{aligned}
$$

where $S, M, H$ refer to the soft, medium and hard bands respectively. If both bands in the hardness ratio contained $>100$ counts, and had an $\mathrm{S} / \mathrm{N}>2$ then the ratios were calculated using the above equations, with the errors on $H$, $S$ and $M$ taken as $\sqrt{\{} H, M, S\}$ respectively and propagated through Equations (13) and (14). For fainter sources we used the Bayesian method of Park et al. (2006), where we used the effective area option in their code to include the count-rate correction factors in the calculation. While the Bayesian method gives asymmetric errors (which are typically a few percent larger than the standard method returns), the standard method returns symmetric errors. This means one can find, for example, HR1 $=0.95 \pm 0.1$, even though the HR must be between -1 and 1 (inclusive). In such cases of course, the true HR limit is +1 (or -1 in a negative counterexample).

\subsection{Manual Screening}

While the quality flagging system based on the source likelihood values is reliable for celestial point sources, it can be deceived in the presence of structured diffuse emission (e.g., from a supernova remnant) or irumental artifacts. The most common of these artifacts is stray light (Moretti et al. 2009): $\mathrm{X}$-ray photons from a source $35^{\prime}-75^{\prime}$ off-axis (i.e., outside the field of view) that are directed onto the XRT detector via a single reflection (as opposed to the double reflection which focuses $\mathrm{X}$-rays). This occurs at a very low level: the effective area of the XRT for a source $0^{\prime}$ off-axis is $\sim 33,000$ times lower than that on axis, and the singly reflected photons are distributed over a much wider area of the CCD than for a focused source. Nonetheless, sufficiently bright sources outside the field of view can cause concentric arcs of events to be detected in the CCD (Figure 5) which can give rise to spurious source detections.

The typical background level of the observations in our catalog is $\sim 10^{-6}$ counts $\mathrm{s}^{-1}$ pixel $^{-1}$; for a source outside the field of view to contribute stray light at this level it would require an on-axis XRT count rate of $\sim 3$ counts $\mathrm{s}^{-1}$. We conservatively chose a limit of 1 counts $\mathrm{s}^{-1}$, which corresponds to a $0.3-10 \mathrm{keV}$ flux of $3.5 \times 10^{-11} \mathrm{erg} \mathrm{cm}^{-2} \mathrm{~s}^{-1}$ assuming a typical AGN spectrum: a power-law spectrum with $N_{\mathrm{H}}=3 \times 10^{20} \mathrm{~cm}^{-2}$ and $\Gamma=1.7$. We identified all sources in the Rosat PSPC and 2XMMi-DR3 catalogs with fluxes above this limit, and selected for manual screening all fields in our catalog that lay within $28^{\prime}-82^{\prime}$ of those sources. This did not identify all fields affected by stray light, as $2 \mathrm{XMMiDR} 3$ covers only a small fraction of the sky, Rosat is not sensitive to strongly absorbed or hard sources and some objects are variable.

There are other artifacts that can contaminate the images. These are residual bright Earth contamination, the "ring of fire" effect caused by serious optical loading (Figure 5, bottom) and the presence of extended sources or diffuse emission. All of these effects (and stray light) give rise to spatially proximate spurious detections. For this reason we also selected for manual screening any image where the median distance between detections was $<80^{\prime \prime}$. In total 15,152 datasets (out of 56,275 in the catalog) were selected for human inspection.

We inspected these images in decreasing order of exposure time. If an image was deemed to be affected by the artifacts

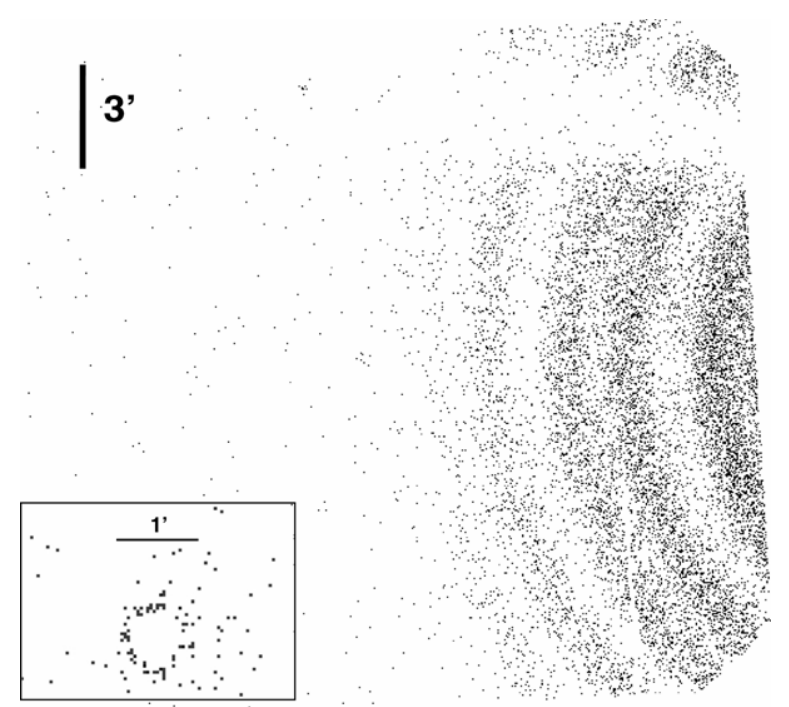

Figure 5. Examples of artifacts that were identified by manual screening. The main plot shows stray light: caused by single reflections from a bright source lying outside the XRT field of view-in this case the Crab nebula, lying 45' off-axis. The gap in the rings is the shadow of the mirror support structure. Inset: a "ring of fire": the apparent X-ray events were caused by accumulation of optical photons from a bright star ( $\mathrm{a} V=3 \mathrm{Be}$ star in this example). Toward the center of the star's location no events are detected because the optical flux is so high that in a single $2.5 \mathrm{~s}$ CCD exposure frame all pixels register events, and thus the event "grade" (which describes how many pixels given event affected) is above the maximum value permitted for valid events.

described above, then the results of this screening was applied to all pointings covering that location on the sky, avoiding the need to check each image individually. When artifacts were manually identified, regions were defined which encompassed them, and any sources which lay within those regions had their detection flags changed. The "Field flag" for the image was also set from its default value of Good $(=0)$ to Flagged $(=1$ or 2$)$. For images containing artifacts (stray light, bright Earth or rings of fire) the detection flag of affected sources was increased (from 0,1 or 2) by 8 and the field flag set to 1 . For images containing diffuse emission the detection flag of affected sources was increased by 16 and the field flag set to 2 .

We distinguish between artifacts and diffuse emission because, while both of these phenomena affect the background map (by causing inhomogeneities over which the background map attempts to smooth and interpolate, and potentially by causing the detection of spurious sources which in turn are added into the background map), artifacts have well defined edges, but it is often not clear where a diffuse source stops contributing to the background. For this reason (given that a dataset can only have a single field flag value) where both artifacts and diffuse emission were identified in an image, the flag was set for the latter.

The result of the screening is that any source with a detection flag with a value $\geqslant 8$ (i.e., lying inside a region which has been manually marked as contaminated) has a high probability of being spurious, whereas sources with a flag value below this but lying in a Flagged field (i.e., in the field, but outside the region manually marked as bad) have false positive rates as described in Section 3.4, but may have incorrect background values and thus measured source fluxes.

\subsection{Astrometric Corrections}

We attempted to derive a more accurate astrometric solution for our datasets than that available from the star 
trackers mounted on the XRT. The latter gives positions accurate to 3..5 $90 \%$ of the time (Moretti et al. 2007). For each dataset, we matched the Good and Reasonable sources with the Two Micron All Sky Survey (2MASS) catalog (Skrutskie et al. 2006) using an approach similar to that employed by Butler (2007). For every dataset in which more than two X-ray sources were detected, we retrieved a list of 2MASS objects that lay within the XRT field of view and attempted to find an aspect solution for the field which maximized the likelihood:

$$
L=\sum_{\mathrm{ox}<20} e^{\left(-0.5 \delta^{2} / \sigma^{2}\right)},
$$

where $o x$ is the angular separation between each Good and Reasonable XRT source and each 2MASS source, so the sum is over all XRT/2MASS source pairs within $20^{\prime \prime}$ of each other; $\delta$ is the angular distance between the 2MASS and XRT sources in question, and $\sigma$ is the radial uncertainty in the two positions added in quadrature. The $1 \sigma$ uncertainty in the aspect solution thus derived was taken as the rms of the $\delta$ value for each 2MASS/XRT pair in the final fit. If the mean shift in any of the X-ray positions as a result of this process was $>15^{\prime \prime}$ then the solution was considered unreliable and rejected: this distance corresponds to a $7 \sigma$ inaccuracy in the star tracker solution, which is a most unlikely situation.

This process could not find an astrometric solution for every dataset, and in the majority of cases where a solution was found, the uncertainty in the aspect solution was $>3$.'.5; in these cases we used the star tracker attitude. A solution with an error $<3$.'.5 was found for only $4 \%$ of the datasets in our catalog, but as these were the datasets with objects in them, $26 \%$ of the sources in our final catalog have positions improved using this technique. Whichever method was used, the astrometric error was added in quadrature to the statistical position error from the PSF fit (Section 3.3.4) to give the radial position error reported in the catalog.

To verify that this method gives reliable positions and uncertainties, we applied it to the fields containing the 999 objects in our catalog which are within $20^{\prime \prime}$ of quasars in the Sloan Digital Sky Survey (SDSS) Quasar Catalog DR5 (Schneider et al. 2007) and thus likely to be the X-ray counterpart to the quasar. We found that $90 \%$ of the XRT positions thus produced agreed with the SDSS positions at the $90 \%$ confidence level, as expected.

\subsection{Building the Final Unique Source List}

Once the above steps had been completed for every dataset contributing to the catalog, we merged the lists of sources from each dataset into one final source list. This was done in the same way as described in Section 3.5, except that instead of using fixed merge radii based on the source brightness, different detections were assumed to be the same source if their positions agreed at the $99.99999426 \%$ level. ${ }^{17}$ For a typical source this was $\sim 14^{\prime \prime}$ ( $\sim 6$ XRT pixels), which is $75 \%$ of the PSF FWHM and the probability of distinct sources lying this close to each other is very low, $\ll 1 \%$.

\footnotetext{
17 i.e., the $5 \sigma$ level of a Gaussian distribution. Since radial errors follow a Rayleigh distribution, we use the probability level, not the number of $\sigma$. By "the positions agreed" at this level we mean that the probability from Rayleigh statistics of their separation being that observed or lower, given their position errors, is less than this threshold.
}
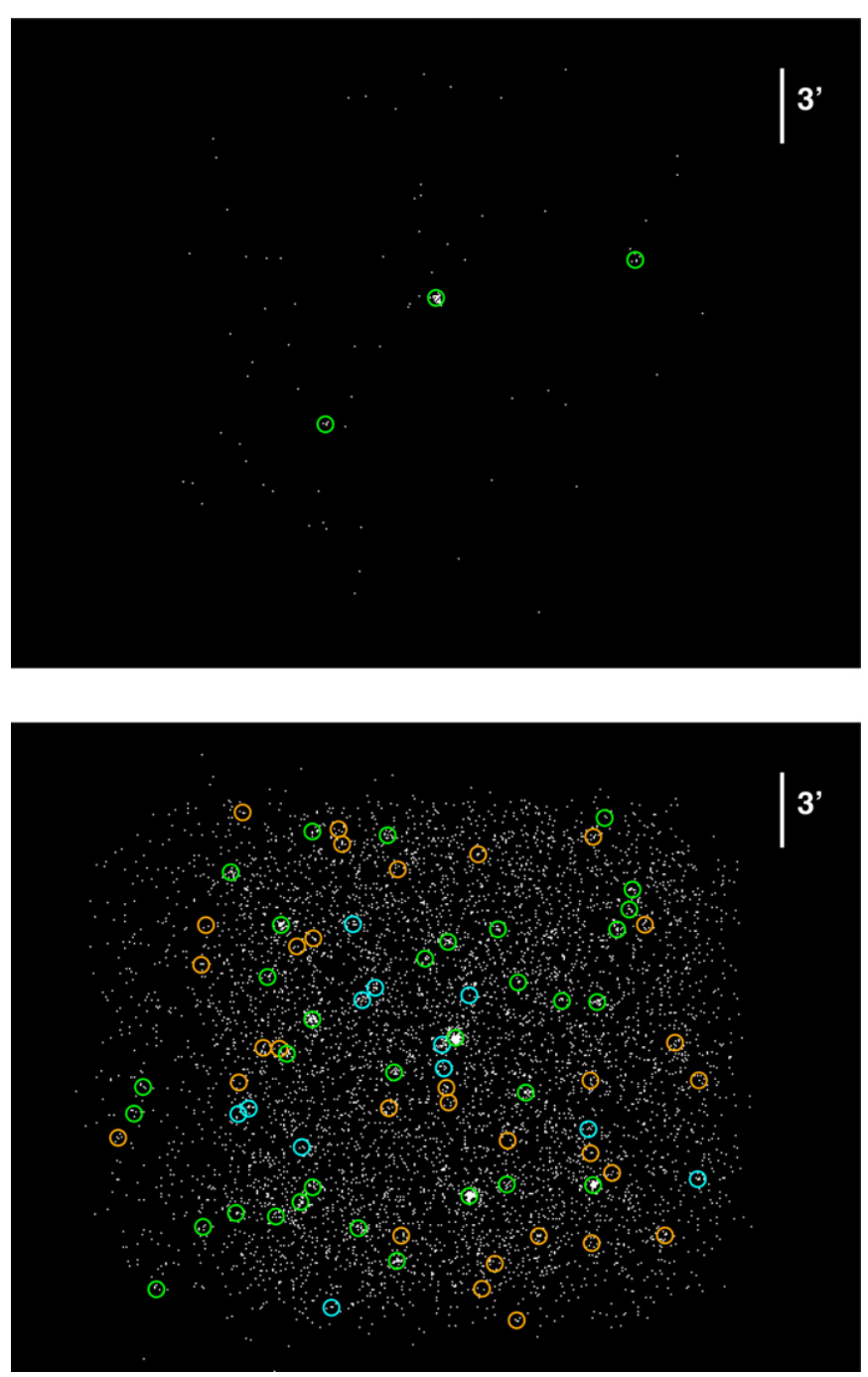

Figure 6. Example datasets from the catalog. Both images are from the total band $(0.3-10 \mathrm{keV})$ with pixel intensity following a log scale. Top: a short single observation, (ObsID 00032165001, exposure $424 \mathrm{~s}$ ) with the three sources detected in that observation and band shown. Bottom: a deep stacked image (field 7086, exposure 1.1 Ms); the final unique source list for this region is shown. The regions indicate objects detected, with the "quality" of the detection shown by the color: green $=$ Good, cyan $=$ Reasonable, orange $=$ Poor The regions are a fixed size and do not reflect the size of the region used in source detection.

This approach takes into account the fact that different observations may have different astrometric accuracy, and allows for faint sources that are near to a bright source, but not detected until after that object has faded, to be distinguished from the bright source. When compiling this final source list, the detection flag in each band was set to the best of the detection flags in that band from the individual detections of the source. A final, overall detection flag was also produced which was the best of the per-band flags, and likewise for the field flag. The optical loading warning was set to be the worst value from the set of individual detections of the source. The final source position was taken from the detection with the smallest position error, and the source was given a unique designation of the form: 1SXPS JHHMMSS.S+DDMMSS. This acronym has been registered with the IAU.

Figure 6 shows two examples of datasets after all of the steps in this section have been applied. 


\section{SOURCE-SPECIFIC PRODUCTS}

The details of the unique sources and the individual detections are available in the form of catalog tables, available to query online or and download (Section 5). In addition to these, we have produced light curves, hardness ratios and variability and flux estimates for each source, and spectra for the brightest sources, as described below. These products are available to download via the 1SXPS Web site, where tools also exist to calculate upper limits for specific locations on the sky.

\subsection{Temporal Products}

We produced light curves in each of the four energy bands, with one bin per observation and one bin per snapshot (for observations where the source is undetected the latter light curve only contains a single bin integrated over that observation). We also produced time series of the hardness ratios with one bin per observation. The times of each bin in all of these products were corrected to the solar system barycenter (i.e., TDB).

To construct the time series, the count rate in each snapshot or observation was determined as described in Section 3.3.4, except that we used the best source position determined per observation (see Section 3.5), to account for the potential differences in astrometry between observations. The sourcecount accumulation region used was also that from Section 3.5 if the source was detected; for bands, snapshots or observations where the source was not detected a circular region of radius 12 pixels (28.'3) was used.

For the time series in each band we calculated used the Pearson's $\chi^{2}$ (Pearson 1900) to determine the probability that the source was variable. The Pearson's $\chi^{2}$ is defined as:

$$
\chi^{2}=\sum_{i}\left\{\frac{\left(D_{i}-M_{i}\right)^{2}}{M_{i}}\right\},
$$

where $D$ and $M$ are the data and model in bin $i$ respectively. These must be not in units of the count rate (as contained in the light curve), but the measured number of counts $(C)$ in each bin. Since we test for the null hypothesis that the source is constant, the model is that of constant source flux, but this is not the same as constant source counts in each bin as the exposure time $(E)$ and count-rate correction factor ( $\kappa$, see Section 3.3.4) can vary from bin to bin. Explicitly including these factors and the background level, if the source is constant the count rate is the same in each bin and is simply the mean value; which can be determined from the measurements thus:

$$
\begin{aligned}
R_{i} & =\text { const } \\
& =\frac{\left(C_{i}-B_{i}\right) \kappa_{i}}{E_{i}} \\
& =\frac{\sum_{j}\left\{\kappa_{j}\left(C_{j}-B_{j}\right)\right\}}{E_{\mathrm{tot}}},
\end{aligned}
$$

where the summation is over all bins, and gives the total number of PSF-corrected counts over the light curve. We can then solve the above to determine the model of the number of counts per bin:

$$
\begin{aligned}
M_{i} & =C_{i} \\
& =\left(\frac{E_{i}}{E_{\mathrm{tot}}}\right)\left(\frac{\sum_{j}\left\{\kappa_{j}\left(C_{j}-B_{j}\right)\right\}}{\kappa_{i}}\right)+B_{i}
\end{aligned}
$$

This test, which reports the probability of the null hypothesis that the source is constant, was applied to both the per-snapshot and per-observation light curves (but not the hardness ratio time series), probing variability on multiple timescales. To ensure that the per-snapshot result is not affected by variation on the per-observation timescale, we calculated $\chi^{2}$ and hence $P$ for the per-snapshot light curve of each observation independently, and then report the lowest value thus obtained.

We also tried using the Wald-Wolfowitz runs test (Wald $\&$ Wolfowitz 1940) as an independent measure of variability, however this lacked the power to identify variable sources in our catalog, probably because many light curves have small numbers of bins. We therefore elected not to include these results in the catalog.

\subsection{Flux Conversions and Spectra}

For every source in the catalog we created energy conversion factors (ECFs) to convert from count rate to flux (observed and unabsorbed $)^{18}$ We did this for two commonly observed spectral types: an absorbed power-law and an absorbed APEC optically thin thermal plasma model (Smith et al. 2001); for the latter we assumed solar abundances. The absorption was modeled using the TBABs model (Wilms et al. 2000).

For each source in the catalog we first determined ECFs using standardized spectra: a power-law with a photon index of 1.7, and an APEC with a temperature of $1 \mathrm{keV}$; the absorption was fixed at the Galactic value in the direction of the source, determined using the Nнтот tool of Willingale et al. (2013).

We also estimated the flux and spectral parameters from the hardness ratio information. Using XSPEC we simulated a series of spectra, with $17 \leqslant \log N_{\mathrm{H}} /\left(1 \mathrm{~cm}^{-2}\right) \leqslant 24$; for the power-law spectrum we used photon indices in the range $-3 \leqslant \Gamma \leqslant 5$ and for the APEC spectrum we used temperatures $-2 \leqslant$ $\log (k T / 1 \mathrm{keV}) \leqslant 1.9$. We folded each simulated spectrum through the instrument response to derive its ECF and its two hardness ratios. We used the latter to construct a look-up table of the spectral parameters as a function of (HR1, HR2); examples are given in Figure 7. For each source in our catalog, if (HR1, HR2) lay in the region covered by the simulated spectra we interpolated on this grid to ascertain the spectral parameters of the source. We also did this for the four points given by $\left(\mathrm{HR} 1 \pm \sigma_{\mathrm{HR} 1}, \mathrm{HR} 2 \pm \sigma_{\mathrm{HR} 2}\right)$ to estimate the uncertainty on these properties. For any of those limits which lay outside the range covered by the simulated spectra, we took the values for the (HR1, HR2) point nearest to the limit in question. Note that the range of parameters for the simulated spectra goes beyond what we may physically expect for XRT sources, in such extreme cases the purpose of this approach is to give reasonable flux estimates within the $0.3-10 \mathrm{keV}$ band, over which the model gives an acceptable approximation to the data. However the actual the spectral parameters themselves should be viewed with caution in those cases, and care should be used before extrapolating outside of the XRT bandpass.

For sources where (HR1, HR2) lay outside the range covered by the simulated spectra we cannot calculate the spectral parameters in this way, instead we determined the probability of measuring (HR1, HR2) if the true spectrum were that of the simulated spectrum with hardness ratios closest to the measured values, given the uncertainties on those values.

\footnotetext{
18 The uncertainty in the ECF was not propagated into the error on the flux;
} this was simply the count-rate error multiplied by the ECF. 

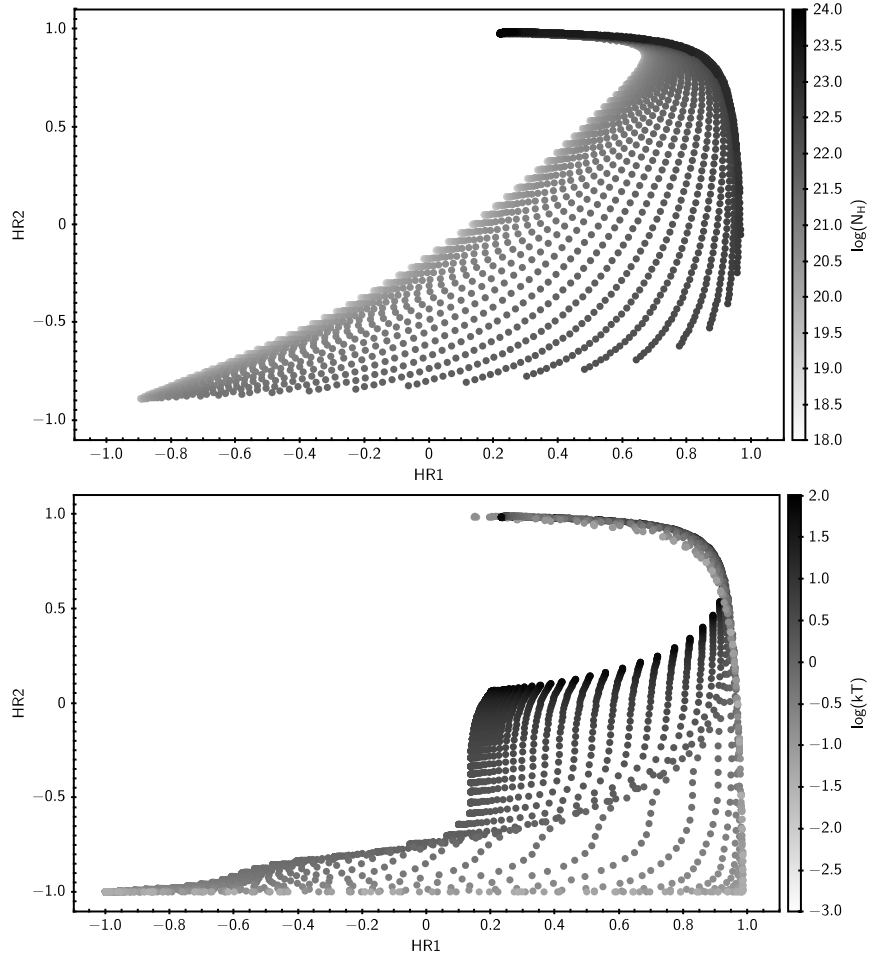

Figure 7. (HR1, HR2) plots used to deduce spectral information for the sources Top: For a power-law spectrum, the $N_{\mathrm{H}}$ values (grayscale) as a function of (HR1, HR2); each point also has a $\Gamma$ value and $E C F$, not shown here. Bottom: For an APEC spectrum, the $k T$ values are shown as the grayscale. Each point also has $N_{\mathrm{H}}$ and ECF values not shown here.

For the sources with at least 50 net events in the total band, we also built spectra using the software of Evans et al. (2009). We fitted these with an absorbed power-law and absorbed APEC, with all parameters unconstrained (i.e., the fitted absorption was independent of the expected Galactic value). The fit was performed on spectra binned to at least one photon per bin (i.e., GROUP MIN 1 in GRPPHA), fitted using the XSPEC $W$-statistic ${ }^{19}$; after fitting we calculated $\chi^{2}$ using the Churazov weighting option (Churazov et al. 1996) to indicate the fit quality and allow users to reject poor fits. Note that this is not a reliable goodness-of-fit indicator (see Churazov et al. 1996, Section 3.2) and cannot be used to calculate the null hypothesis probability.

In the final catalog table we report the spectral properties derived through all three of the above methods (fixed spectra, interpolation of the HR values, and spectral fitting) where they are available. Since not all objects have all of the properties, this can make comparison of sources awkward, we have therefore included in the catalog a set of "best spectral properties." These are taken from the spectral fit if it exists, otherwise the HR interpolation, and if neither of those is available, the results from the fixed spectrum are used here.

\subsection{Cross-correlation with External Catalogs}

We cross-correlated the 1SXPS catalog with various external catalogs and databases, defining a source match to be where the 1SXPS and external catalog positions agree at the $99.7 \%$

\footnotetext{
19 That is, by requesting the $\mathcal{C}$-statistic and then providing a background spectrum with Poisson statistics, see https://heasarc.gsfc.nasa.gov/xanadu/ $\mathrm{xspec/manual/XSappendixStatistics.html.}$
}

level. ${ }^{20}$ SIMBAD $^{21}$ contains some sources from the facilityspecific catalogs that we searched; such sources were only taken from the facility catalogs rather than repeating the match via SIMBAD/NED. We assumed zero position uncertainty for the SIMBAD, USNO-B1, 2MASS, NED and SDSS QSO catalogs, using just the 1SXPS position errors. For the USNO-B1, 2MASS and SDSS catalogs this is because their position errors are negligible compared to the 1SXPS errors. For SIMBAD and NED we are not able to specify the search radius as a function of source error in the remote query, and error information is not available in a uniform way; this may mean that for these catalogs the number of real matches which are not reported is higher than for the other catalogs. For the remaining catalogs we used the 1SXPS and catalog error added in quadrature. In some catalogs the systematic error is given only in the supporting documentation. This was added in quadrature to the catalog's statistical error when available. Details of the catalogs and their systematic errors are given in Table 6, along with the number of 1SXPS sources which have a match in each catalog. Spatial coincidence alone of course does not guarantee association between the 1SXPS source and that in the external catalog. To estimate the number of spurious matches in this correlation, we shifted the position of each 1SXPS source at random by $1^{\prime}-2^{\prime}$ and repeated the correlation test. The number of matches found to these positions are also shown in Table 6.

Due to the high sky density of the 2MASS and USNO-B1 catalogs, the number of expected spurious matches is very high at $>50 \%$. Indeed, there are frequently multiple matches from these catalogs to a single 1SXPS source, indicating that a $3 \sigma$ spatial coincidence in this case it a poor indicator of association.

We therefore ignored matches from these catalogs to estimate the number of new sources in our catalog: we found 68,638 1SXPS objects which are uncataloged (i.e., had no external catalog matches) in this case. However, as Table 6 shows, despite these considerations there are 62,712 objects without a match in the USNO-B1 catalog and 99,353 without a match in the 2MASS source; in total there are 20,390 sources in the 1SXPS catalog with no counterpart in any of the catalogs against which we performed a cross-correlation.

\subsection{Upper Limit Server}

The 1SXPS Web site includes an upper limit server, which allows upper limits to be calculated for any sky location covered by our catalog. If the location was observed in more than one observation, upper limits can be calculated per observation, or from the stacked image in which those observations are included. To calculate the upper limit a 12 pixel radius circle is placed on the image at the location in question, and the number of events in that circle is registered. The background level in this region is taken from the corresponding background map. Then the Bayesian method of Kraft et al. (1991) is used to determine the upper limit on the source count rate at the confidence level specified by the user. If the location requested matches that of a Bad detection which was discarded from the catalog (Section 3.4), this is also reported.

\footnotetext{
20 i.e., the Gaussian " $3 \sigma$ ” level, although as we used Rayleigh statistics we did not use $3 \sigma$, but $99.7 \%$. This is smaller than the search radius used to merge distinct 1SXPS detections in to a unique source list, because the sky density of some external catalogs is high, and the number of spurious associations expected using a " $5 \sigma$ " radius was unacceptably large.

21 The SIMBAD and NED catalogs are dynamic entities: we cross-correlated against SIMBAD on 2013 June 10 and NED on 2013 September 6.
} 
Table 6

Catalogs Cross-correlated with 1SXPS

\begin{tabular}{|c|c|c|c|}
\hline Catalog & Systematic Error $^{\mathrm{a}}$ & Number of Matches ${ }^{b}$ & Spurious Matches $^{\mathrm{c}}$ \\
\hline SDSS Quasar Catalog DR5 ${ }^{\mathrm{d}}$ & & 1,781 & $9(0.5 \%)$ \\
\hline XRTGRB $^{\mathrm{e}}$ & & 659 & $6(1 \%)$ \\
\hline SwiftFT $^{\mathrm{f}}$ & & 9,154 & $268(3 \%)$ \\
\hline 1SWXRT & & 35,009 & $1,669(5 \%)$ \\
\hline $1 \mathrm{CSC}^{\mathrm{h}}$ & & 6,334 & $340(5 \%)$ \\
\hline $3 \mathrm{XMM} \mathrm{DR} 4^{\mathrm{i}}$ & & 19,649 & $1,381(7 \%)$ \\
\hline ROSHRI $^{\mathrm{j}}$ & $10^{\prime \prime}$ & 1,930 & $171(9 \%)$ \\
\hline SIMBAD $^{\mathrm{k}}$ & & 17,708 & $2,000(11 \%)$ \\
\hline $\mathrm{XMM} \mathrm{SL1}^{\mathrm{l}}$ & $17^{\prime \prime}$ & 2,212 & $378(17 \%)$ \\
\hline ROSPSPC $^{\mathrm{m}}$ & $25^{\prime \prime}$ & 4,968 & $1,082(22 \%)$ \\
\hline $\mathrm{NED}^{\mathrm{n}}$ & & 49,098 & $14,761(30 \%)$ \\
\hline USNO-B $1^{\circ}$ & & 88,812 & $48,718(55 \%)$ \\
\hline 2MASSp & & 52,171 & $33,549(64 \%)$ \\
\hline
\end{tabular}

Notes. ${ }^{\text {a }} 90 \%$ confidence. ${ }^{b}$ Number of 1SXPS sources for which there is a counterpart in the external catalog within $3 \sigma{ }^{\mathrm{c}}$ The number of 1SXPS sources with a match after the 1SXPS position has been moved by $1^{\prime}-2^{\prime}$; the value in brackets is this number as a percentage of the matches to 1SXPS positions for the same external catalog. ${ }^{\mathrm{d}}$ Schneider et al. (2007). ${ }^{\mathrm{e}}$ Taken from http://www.swift.ac.uk/xrt_positions; see Evans et al. (2009). ${ }^{\mathrm{f}}$ Puccetti et al. (2011). ${ }^{\mathrm{g}}$ D'Elia et al. (2013). ${ }^{\mathrm{h}}$ Evans et al. (2010). ${ }^{\mathrm{i}}$ http://xmmssc-www.star.le.ac.uk/Catalogue/3XMM-DR4/. $\mathrm{j}$ http://heasarc.gsfc.nasa.gov/W3Browse/rosat/roshri.html. ${ }^{\mathrm{k}}$ http://simbad.u-strasbg.fr/simbad/. ${ }^{1}$ Saxton et al. (2008). ${ }^{\mathrm{m}}$ Voges et al. (1999). ${ }^{\mathrm{n}}$ http://ned.ipac.caltech.edu/. ${ }^{o}$ Monet et al. (2003). ${ }^{\mathrm{p}}$ Skrutskie et al. (2006).

\section{CATALOG CHARACTERISTICS AND AVAILABILITY}

The 1SXPS catalog contains 151,524 sources; 135,086 of which are not in flagged regions (Section 3.6). The median 90\% confidence radial position error of the sources in the full catalog is 5.5 , including systematic errors, and the median $0.3-10 \mathrm{keV}$ flux is $3 \times 10^{-14} \mathrm{erg} \mathrm{cm}^{-2} \mathrm{~s}^{-1}$. The total exposure time of the observations in the catalog is $147 \mathrm{Ms}$, spread over $1905 \mathrm{deg}^{2}$ on the sky. $10 \%$ of the exposure time lies at a Galactic latitude $|b|<3^{\circ} ; 14 \%$ of 1SXPS sources lie in this latitude range, showing as expected an overdensity of sources in the Galactic plane compared to the sky as a whole.

The catalog of sources and their properties is available for download as a FITS or ASCII table from the 1SXPS Web site: http://www.swift.ac.uk/1SXPS. Table 7 describes the columns in the catalog. This Web site also provides simple and comprehensive search facilities, a detailed Web site for each source and each dataset, as well as the upper limit server (Section 4.4). The main catalog file is also available through Vizier (catalog ID: IX/43).

A table of external catalog cross-correlations (Section 4.3) is available from the site above, as are tables giving information about the individual detections and the datasets. These tables are described in Tables $8-10$. We request that publications which make use of this catalog state in the acknowledgements: This work made use of data supplied by the UK Swift Science Data Centre at the University of Leicester as well as citing this paper.

When selecting objects from the tables, the combination of detection flags and field flags gives great control over whether sensitivity or purity is prioritized. The catalog Web site also provides postage-stamp images of each source and images of each dataset; when considering sources with detection flags $\geqslant 8$ it is recommended to view these images to help judge their reliability. For the rest of this paper we conservatively defined a "clean" subsample of the catalog, comprising all objects with detection and field flags both $<2$ (i.e., Good or Reasonable, and from a field that is either OK, or affected only by artifacts but not in the region covered by the artifact): there are 98,762 such sources in the catalog.

\section{VERIFICATION}

We used simulations to verify the accuracy of the catalog, making these as realistic as possible by basing our simulations on real data. To do this we identified XRT observations of 2XMMiDR3 (Watson et al. 2009) fields, selected from that catalog all sources expected to contribute at least two events to the XRT image (assuming a typical AGN spectrum: $N_{\mathrm{H}}=$ $3 \times 10^{20} \mathrm{~cm}^{-2}, \Gamma=1.7$ ), and visually inspected the XRT image to ensure that this list identified all objects in the field. We then passed this source list to our background map software, which created a model of the background in the real XRT image. This model then forms the basis of the simulations. We did this for a range of different positions on the sky and XRT exposure times.

To simulate an image we then used the background map just created, with the corresponding exposure map to measure the number of background counts, $\mu_{i}$, in each pixel $i$. For each pixel in the image we drew the number of events to simulate at random from a Poisson distribution with a mean of $\mu_{i}$. To add sources to the image we randomly drew from the $\log N-\log S$ distribution of extragalactic sources from Mateos et al. (2008). For each source we randomized the position on the CCD, and then simulated $C$ events, where $C$ was drawn randomly from a Poisson distribution with a mean equal to the number of events expected from that source on-axis. These events were folded through the instrumental PSF to locate the specific pixel in which the photon fell. If the exposure map value at this pixel was less than the on-axis exposure value, a random number between 0 and 1 was generated. If this number was less than the fractional exposure of the pixel in question, the photon was added to the image, otherwise it was discarded. In this way we build up a realistic XRT image.

Although we had a discrete set of "seed" images from which we could simulate data, by selectively excluding snapshots from those images, we were able to simulate a larger selection of exposure times than would be given simply by considering the seed images as unit elements. Similarly, we could simulate a range of background levels by multiplying the seed background map by an appropriate value. This allowed us to test our catalog 
Table 7

Contents of the Main Catalog Table ("Sources")

\begin{tabular}{ll}
\hline \hline Field & \\
\hline & \\
Name & Units \\
R.A. & deg \\
Decl. & deg \\
Err90 & arcsec \\
AstromType & \\
$l$ & deg \\
$b$ & deg \\
OffAxis & arcmin \\
& \\
& \\
Exposure & $\mathrm{s}$ \\
StartDate & UT \\
StopDate & UT \\
NumObs & \\
NumDetObs & \\
& \\
DetFlag & \\
Fieldflag & \\
DetFlag_band[0-4] &
\end{tabular}

Rate_band[0-4]

Counts_band[0-4]

BGCounts_band[0-4]

CF_band[0-4]

PvarPchiSnapshot_band[0-4]

PvarPchiObsID_band[0-4]

HR1

HR2

GalNH

$\mathrm{cm}^{-2}$

whichPow

whichAPEC

PowECFO

PowECFU

PowFlux

PowPeakFlux

PowUnabsFlux

PowPeakUnabsFlux

counts s $\mathrm{s}^{-1}$

(1)

nits

(n)

Name and position

Right Ascension (J2000)

Declination (J2000)

90\% conf. radial position error

The provenance of the astrometry used for the source position.

$0=$ Swift star tracker, $1=\mathrm{XRT} / 2 \mathrm{MASS}$ correlation

Galactic longitude

Galactic latitude

The mean off-axis angle of this source from the observations in which it was detected

\section{Exposure details}

The total exposure at the source location

The calendar date of the start of the first observation

of the location of this source

The calendar date of the end of the last observation

of the location of this source

The number of observations of the location of the source

The number of observations in which the source was detected

\section{Flag details}

The best detection flag from all detections of this source

The best field flag from all detections of this source

The best detection flag in each band, from all detections of the source in that band

Count rate and variability information

The mean count rate of the source in each band

The number of counts measured in the region of the source in each band

The number of counts in the background map

in the region of the source in each band

The count-rate correction factor $(\kappa)$ for the source in each band

The probability that the source is constant

between snapshots in band $0-4$,

deduced via the Pearson's $\chi^{2}$ test

The probability that the source is constant

between observations in band $0-4$,

deduced via the Pearson's $\chi^{2}$ test

The HR1 hardness ratio

The HR2 hardness ratio

Flux and spectral information

The Galactic absorption column density in the direction of the source

The provenance of the summary spectral fields for the power-law model

$0=$ fixed spectrum, $1=$ HR-derived, $2=$ fitted spectrum

The provenance of the summary spectral fields for

the APEC model

$0=$ fixed spectrum, $1=\mathrm{HR}$-derived, $2=$ fitted spectrum

Summary spectral information ${ }^{\mathrm{b}}$

$\operatorname{erg} \mathrm{cm}^{-2}$ counts $^{-1}$

$\operatorname{erg~} \mathrm{cm}^{-2}$ counts $^{-1}$

The counts-to-observed-flux energy conversion factor derived from the power-law spectrum

The counts-to-unabsorbed-flux energy conversion factor derived from the power-law spectrum

The mean observed source flux derived

from the power-law spectrum

The peak ${ }^{\mathrm{c}}$ observed source flux derived from the power-law spectrum

The mean unabsorbed source flux derived from the power-law spectrum

The peak ${ }^{\mathrm{c}}$ unabsorbed source flux derived from the power-law spectrum
Yes

Yes

Yes

Yes

Yes

Yes

Yes 
Table 7

(Continued)

\begin{tabular}{|c|c|c|c|}
\hline Field & Units & Description & Has Errors?a \\
\hline APECECFO & 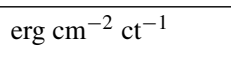 & $\begin{array}{l}\text { The counts-to-observed-flux energy conversion factor } \\
\text { derived from the APEC spectrum }\end{array}$ & \\
\hline APECECFU & 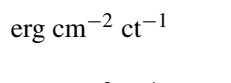 & $\begin{array}{l}\text { The counts-to-unabsorbed-flux energy conversion factor } \\
\text { derived from the APEC spectrum }\end{array}$ & \\
\hline APECFlux & $\operatorname{erg~cm}{ }^{-2} \mathrm{~s}^{-1}$ & $\begin{array}{l}\text { The mean observed source flux derived } \\
\text { from the APEC spectrum }\end{array}$ & Yes \\
\hline APECPeakFlux & $\mathrm{erg} \mathrm{cm}{ }^{-2} \mathrm{~s}^{-1}$ & $\begin{array}{l}\text { The peak }{ }^{\mathrm{c}} \text { observed source flux derived from the } \\
\text { APEC spectrum }\end{array}$ & Yes \\
\hline APECUnabsFlux & 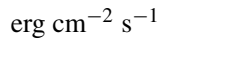 & $\begin{array}{l}\text { The mean unabsorbed source flux derived from the } \\
\text { APEC spectrum }\end{array}$ & Yes \\
\hline APECPeakUnabsFlux & 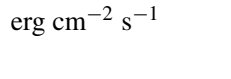 & $\begin{array}{l}\text { The peak }{ }^{c} \text { unabsorbed source flux derived from the } \\
\text { power-law spectrum }\end{array}$ & Yes \\
\hline \multicolumn{4}{|c|}{ Detailed spectral information } \\
\hline FixedPowECFO & 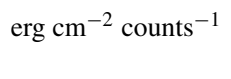 & $\begin{array}{l}\text { The counts-to-observed-flux energy conversion factor } \\
\text { derived from the fixed power-law spectrum }\end{array}$ & \\
\hline FixedPowECFU & erg $\mathrm{cm}^{-2}$ counts $^{-1}$ & $\begin{array}{l}\text { The counts-to-unabsorbed-flux energy conversion factor } \\
\text { derived from the fixed power-law spectrum }\end{array}$ & \\
\hline FixedPowFlux & $\operatorname{erg~cm}{ }^{-2} \mathrm{~s}^{-1}$ & $\begin{array}{l}\text { The mean observed source flux derived } \\
\text { from the fixed power-law spectrum }\end{array}$ & Yes \\
\hline FixedPowUnabsFlux & 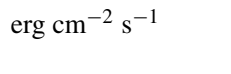 & $\begin{array}{l}\text { The mean unabsorbed source flux derived } \\
\text { from the fixed power-law spectrum }\end{array}$ & Yes \\
\hline FixedAPECECFO & erg $\mathrm{cm}^{-2}$ counts $^{-1}$ & $\begin{array}{l}\text { The counts-to-observed-flux energy conversion factor } \\
\text { derived from the fixed APEC spectrum }\end{array}$ & \\
\hline FixedFixed APECECFU & erg cm ${ }^{-2}$ counts $^{-1}$ & $\begin{array}{l}\text { The counts-to-unabsorbed-flux energy conversion factor } \\
\text { derived from the fixed APEC spectrum }\end{array}$ & \\
\hline FixedAPECFlux & 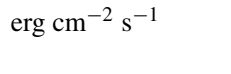 & $\begin{array}{l}\text { The mean observed source flux derived } \\
\text { from the fixed APEC spectrum }\end{array}$ & Yes \\
\hline FixedAPECUnabsFlux & 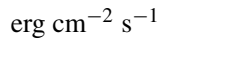 & $\begin{array}{l}\text { The mean unabsorbed source flux derived } \\
\text { from the fixed APEC spectrum }\end{array}$ & Yes \\
\hline InterpPowECFO & erg cm ${ }^{-2}$ counts $^{-1}$ & $\begin{array}{l}\text { The counts-to-observed-flux energy conversion factor } \\
\text { derived from the HR-derived power-law spectrum }\end{array}$ & \\
\hline InterpPowECFU & erg $\mathrm{cm}^{-2}$ counts $^{-1}$ & $\begin{array}{l}\text { The counts-to-unabsorbed-flux energy conversion factor } \\
\text { derived from the HR-derived power-law spectrum }\end{array}$ & \\
\hline InterpPowFlux & 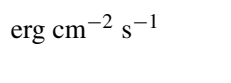 & $\begin{array}{l}\text { The mean observed source flux derived from the HR-derived } \\
\text { power-law spectrum }\end{array}$ & Yes \\
\hline InterpPowUnabsFlux & 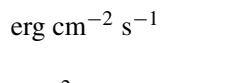 & $\begin{array}{l}\text { The mean unabsorbed source flux derived } \\
\text { from the HR-derived power-law spectrum }\end{array}$ & Yes \\
\hline InterpPowNH & $\mathrm{cm}^{-2}$ & $\begin{array}{l}\text { The absorption column density derived from the } \\
\text { HR-derived power-law spectrum }\end{array}$ & Yes \\
\hline InterpPowGamma & erg & $\begin{array}{l}\text { The power-law photon index derived from the } \\
\text { HR-derived power-law spectrum }\end{array}$ & Yes \\
\hline InterpAPECECFO & $\mathrm{erg} \mathrm{cm}^{-2}$ counts $^{-1}$ & $\begin{array}{l}\text { The counts-to-observed-flux energy conversion } \\
\text { factor derived from the HR-derived APEC spectrum }\end{array}$ & \\
\hline InterpAPECECFU & $\mathrm{erg} \mathrm{cm}^{-2}$ counts $^{-1}$ & $\begin{array}{l}\text { The counts-to-unabsorbed-flux energy conversion } \\
\text { factor derived from the HR-derived APEC spectrum }\end{array}$ & \\
\hline InterpAPECFlux & $\operatorname{erg~cm}{ }^{-2} \mathrm{~s}^{-1}$ & $\begin{array}{l}\text { The mean observed source flux derived } \\
\text { from the HR-derived APEC spectrum }\end{array}$ & Yes \\
\hline InterpAPECUnabsFlux & $\operatorname{erg~cm}{ }^{-2} \mathrm{~s}^{-1}$ & $\begin{array}{l}\text { The mean unabsorbed source flux derived } \\
\text { from the HR-derived APEC spectrum }\end{array}$ & Yes \\
\hline InterpAPECNH & $\mathrm{cm}^{-2}$ & $\begin{array}{l}\text { The absorption column density derived } \\
\text { from the HR-derived APEC spectrum }\end{array}$ & Yes \\
\hline InterpAPECkT & $\mathrm{keV}$ & $\begin{array}{l}\text { The plasma temperature derived from } \\
\text { the HR-derived APEC spectrum }\end{array}$ & Yes \\
\hline P_pow & & $\begin{array}{l}\text { For sources without an HR-derived value, the probability of } \\
\text { measuring the (HR1, HR2) value of this source } \\
\text { if it had an power-law spectrum }\end{array}$ & \\
\hline P_APEC & & $\begin{array}{l}\text { For sources without an HR-derived value, the probability } \\
\text { of measuring the (HR1, HR2) value of this sourc } \\
\text { e if it had an APEC spectrum }\end{array}$ & \\
\hline FittedPowECFO & $\mathrm{erg} \mathrm{cm}^{-2}$ counts $^{-1}$ & $\begin{array}{l}\text { The counts-to-observed-flux energy conversion factor } \\
\text { derivedfrom the fitted power-law spectrum }\end{array}$ & \\
\hline FittedPowECFU & 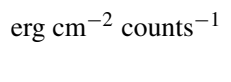 & $\begin{array}{l}\text { The counts-to-unabsorbed-flux energy conversion factor } \\
\text { derived from the fitted power-law spectrum }\end{array}$ & \\
\hline FittedPowFlux & 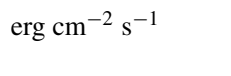 & $\begin{array}{l}\text { The mean observed source flux derived } \\
\text { from the fitted power-law spectrum }\end{array}$ & Yes \\
\hline
\end{tabular}


Table 7

(Continued)

\begin{tabular}{|c|c|c|c|}
\hline Field & Units & Description & Has Errors? ${ }^{\mathrm{a}}$ \\
\hline FittedPowUnabsFlux & 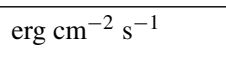 & $\begin{array}{l}\text { The mean unabsorbed source flux derived } \\
\text { from the fitted power-law spectrum }\end{array}$ & Yes \\
\hline FittedPowNH & $\mathrm{cm}^{-2}$ & $\begin{array}{l}\text { The absorption column density derived } \\
\text { from the fitted power-law spectrum }\end{array}$ & Yes \\
\hline FittedPowGamma & erg & $\begin{array}{l}\text { The power-law photon index derived } \\
\text { from the fitted power-law spectrum }\end{array}$ & Yes \\
\hline FittedPowChi & & $\chi^{2}$ of the power-law spectral fit & \\
\hline FittedPowDOF & & Degrees of freedom in the power-law spectral fit & \\
\hline FittedPowRedChi & & $\chi_{v}^{2}$ in the power-law spectral fit & \\
\hline FittedAPECECFO & erg cm ${ }^{-2}$ counts $^{-1}$ & $\begin{array}{l}\text { The counts-to-observed-flux energy conversion factor } \\
\text { derived from the fitted APEC spectrum }\end{array}$ & \\
\hline FittedAPECECFU & erg cm ${ }^{-2}$ counts $^{-1}$ & $\begin{array}{l}\text { The counts-to-unabsorbed-flux energy conversion factor } \\
\text { derived from the fitted APEC spectrum }\end{array}$ & \\
\hline FittedAPECFlux & 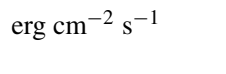 & $\begin{array}{l}\text { The mean observed source flux derived } \\
\text { from the fitted APEC spectrum }\end{array}$ & Yes \\
\hline FittedAPECUnabsFlux & 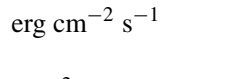 & $\begin{array}{l}\text { The mean unabsorbed source flux derived } \\
\text { from the fitted APEC spectrum }\end{array}$ & Yes \\
\hline FittedAPECNH & $\mathrm{cm}^{-2}$ & $\begin{array}{l}\text { The absorption column density derived } \\
\text { from the fitted APEC spectrum }\end{array}$ & Yes \\
\hline FittedAPECkT & $\mathrm{keV}$ & $\begin{array}{l}\text { The plasma temperature derived from the } \\
\text { fitted APEC spectrum }\end{array}$ & Yes \\
\hline FittedAPECChi & & $\chi^{2}$ of the APEC spectral fit & \\
\hline $\begin{array}{l}\text { FittedAPECDOF } \\
\text { FittedAPECRedChi }\end{array}$ & & $\begin{array}{l}\text { Degrees of freedom in the APEC spectral fit } \\
\chi_{v}^{2} \text { in the APEC spectral fit }\end{array}$ & \\
\hline \multicolumn{4}{|c|}{ Cross-correlation information $^{\mathrm{b}}$} \\
\hline Numxcorr & & The number of matches in the external catalogs & \\
\hline Numxcorr_slim & & $\begin{array}{l}\text { The number of matches in the external catalogs, } \\
\text { excluding USNO-B } 1 \text { and } 2 \text { MASS }\end{array}$ & \\
\hline isROSHRI & & $\begin{array}{l}\text { Whether the object does (1) or does not ( } 0) \text { match } \\
\text { an object in the Rosat HRI catalog }\end{array}$ & \\
\hline isROSPSPC & & $\begin{array}{l}\text { Whether the object does (1) or does not }(0) \text { match } \\
\text { an object in the Rosat PSPC catalog }\end{array}$ & \\
\hline is $3 \mathrm{XMM}$ & & $\begin{array}{l}\text { Whether the object does (1) or does not ( } 0 \text { ) match } \\
\text { an object in the } 3 \text { XMM DR4 catalog }\end{array}$ & \\
\hline isXMMSL1 & & $\begin{array}{l}\text { Whether the object does (1) or does not ( } 0 \text { ) match } \\
\text { an object in the XMMSL1 XMM-Newton Slew Survey }\end{array}$ & \\
\hline isSwiftFT & & $\begin{array}{l}\text { Whether the object does (1) or does not ( } 0) \text { match } \\
\text { an object in the Swift-FT catalog }\end{array}$ & \\
\hline is $1 \mathrm{SWXRT}$ & & $\begin{array}{l}\text { Whether the object does (1) or does not }(0) \text { match } \\
\text { an object in the 1SWXRT catalog }\end{array}$ & \\
\hline isXRTGRB & & $\begin{array}{l}\text { Whether the object does (1) or does not }(0) \text { match } \\
\text { a cataloged XRT position of a gamma-ray burst }\end{array}$ & \\
\hline isSDSSQSO & & $\begin{array}{l}\text { Whether the object does (1) or does not ( } 0) \text { match } \\
\text { an object in the SDSS QSO DR } 5 \text { catalog }\end{array}$ & \\
\hline is 2 MASS & & $\begin{array}{l}\text { Whether the object does (1) or does not }(0) \text { match } \\
\text { a } 2 \text { MASS source }\end{array}$ & \\
\hline isUSNOB1 & & $\begin{array}{l}\text { Whether the object does (1) or does not }(0) \text { match } \\
\text { a USNO-B1 source }\end{array}$ & \\
\hline isSIMBAD & & $\begin{array}{l}\text { Whether the object does (1) or does not }(0) \text { match } \\
\text { a SIMBAD object }\end{array}$ & \\
\hline xcorrIDs & & $\begin{array}{l}\text { A semi-colon delimited list of the identifiers } \\
\text { of the matched sources }\end{array}$ & \\
\hline
\end{tabular}

Notes.

a This is "no" unless stated. For a field with errors, there are two error fields, fieldname_pos and fieldname_neg.

$\mathrm{b}$ This is taken from the detailed spectral information, for the method given in the whichPow and whichAPEC fields.

${ }^{c}$ The peak flux is derived using the summary ECF and the count rate in of the brightest bin in the total band per-snapshot light curve.

software on a range of exposure times and background levels which mirrors that of the data in the catalog.

\subsection{Background Maps}

To confirm that our background mapping was working correctly we simulated 400 images, with the background level and exposure time drawn at random from the distribution of those values seen in the catalog. Since these contain no sources, the true background level of each image can be measured directly. We then used our software to build a background map of these images and measured the background level from these maps, to compare with the true value. We measured the 
Table 8

Contents of the "Datasets" Catalog Table

\begin{tabular}{|c|c|c|}
\hline Field & Units & Description \\
\hline ID & & $\begin{array}{l}\text { The unique identifier of the dataset. For observations } \\
\text { this is the 11-digit ObsID. For stacked images } \\
\text { it is the number of the image }\end{array}$ \\
\hline R.A. & $\operatorname{deg}$ & Right Ascension of the field center (J2000) \\
\hline Decl. & deg & Declination of the field center (J2000) \\
\hline$l$ & $\operatorname{deg}$ & The Galactic longitude of the field center \\
\hline$b$ & deg & The Galactic latitude of the field center \\
\hline IsStacked & & Indicates whether this is a stacked image (1) or not (0) \\
\hline Exposure & $\mathrm{s}$ & The exposure time in the dataset \\
\hline FieldBG_band[0-4] & counts s ${ }^{-1}$ pixel $^{-1}$ & The mean background level in each band \\
\hline Numsrc_band[0-4] & & The number of sources in this image in each band \\
\hline NumOK_band[0-4] & & The number of Good and Reasonable sources in each band \\
\hline MedianNNDist_band[0-4] & & The median distance between the sources in each band's image \\
\hline Date_start & UT & The calendar date of the observation start \\
\hline Date_stop & UT & The calendar date of the observation end \\
\hline FieldFlag & & The field flag \\
\hline NumSnapshots & & The number of snapshots in the dataset \\
\hline AstromErr & $\operatorname{arcsec}$ & $\begin{array}{l}\text { The } 90 \% \text { confidence uncertainty in the astrometric solution } \\
\text { for this field derived using 2MASS (Section 3.7) }\end{array}$ \\
\hline StackedImage & & $\begin{array}{l}\text { For observations: the ID of the stacked image in which this observation is included. } \\
\text { For stacked images: the IDs of any stacked images } \\
\text { which overlap this one }\end{array}$ \\
\hline
\end{tabular}

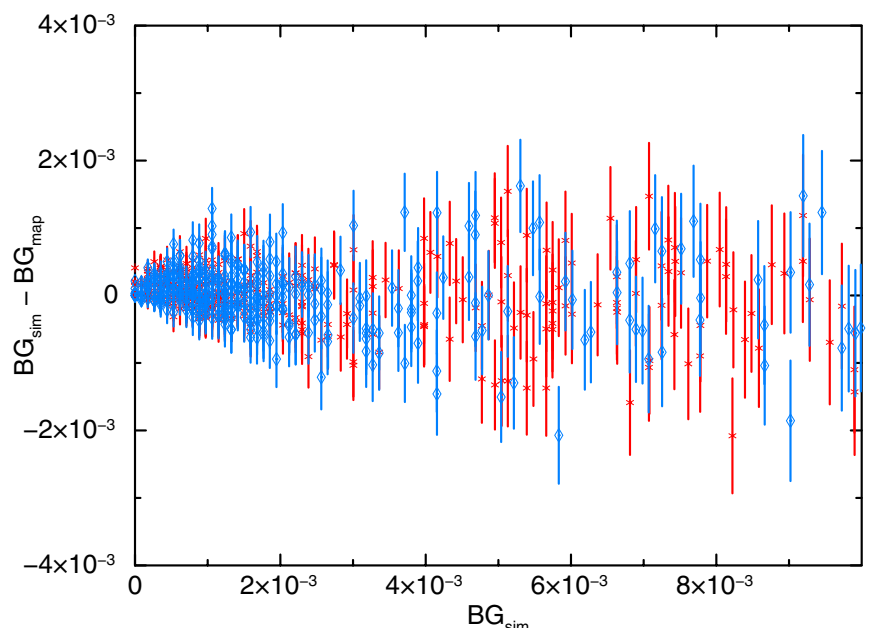

Figure 8. Comparison of the background measured directly from the simulated image $\left(\mathrm{BG}_{\mathrm{sim}}\right)$ with that measured from a background map $\left(\mathrm{BG}_{\mathrm{map}}\right)$ constructed from the simulated image. The red stars show the simulations with no sources included, the blue diamonds the simulations containing a source.

background by placing a circle of radius 60 pixels at a random location on the image and taking the mean value of all pixels in this circle with non-zero exposure. The same circle was used for an image and the corresponding background map, but a different circle was randomly placed for each simulation. The 60 pixel radius is much larger than the source extraction region used in the catalog, but is needed to reduce the magnitude of the Poisson uncertainty on the measurement of the simulated image. Figure 8 shows the results of these tests, confirming that the background mapping tool performs well.

We performed a further 400 simulations independent of the set used above. This time a single source was added to the simulated image, although we also saved the source-less image, from which we measured the true background level. We then ran our source detection code on the image including the source. This detected the source and built a map of the underlying background. As Figure 8 shows, the reconstructed background in these cases still accurately reflects the true value: a $\chi^{2}$ test for the model $\mathrm{BG}_{\text {sim }}-\mathrm{BG}_{\text {map }}=0$ applied to these data gives $\chi_{v}^{2}=$ 0.84 , for 788 dof.

\subsection{Count-rate Reconstruction}

To test whether the source count rate was adequately reconstructed, we performed a further 5,000 simulations, this time with multiple sources per image, as described in Section 6. For each source we drew the flux from the $\log N-\log S$ distribution, multiplied it by the image exposure time and folded it though a typical AGN spectrum to obtain the expected number of XRT events, $\mathcal{T}_{c}$. To incorporate Poisson processes we then drew a number $\mathcal{S}_{c}$ from a Poisson distribution with a mean of $\mathcal{T}_{c}$; this $\left(\mathcal{S}_{c}\right)$ was the number of events which were actually put into the simulation. These events are folded through the PSF and exposure map (Section 6); the number which are actually included in the simulated image is $A_{c}$. Each of these numbers $\left(\mathcal{T}_{c}, \mathcal{S}_{c}, A_{c}\right)$ can be converted to a count rate $(\mathcal{T}, \mathcal{S}, A)$ by dividing by the on-axis exposure time of the simulated image.

We ran the catalog software on these 5000 simulated images to detect and characterize the sources, and then compared the count rates thus obtained with the simulated count rates. The top panel of Figure 9 shows the distribution of $(R-\mathcal{S}) / \sigma_{R}$, where $R$ and $\sigma_{R}$ are the source count rate and error returned by the catalog software. This shows that our software is accurately reconstructing the count rates. The non-zero width of the distribution arises because of the PSF corrections and Poisson noise: if a source is located on the detector such that, on average, $30 \%$ of the simulated events are lost (i.e., $A / \mathcal{S}=0.7$ ) then the catalog software (correctly) applies a correction of $\kappa=1 / 0.7$ to the measured count rate. However due to Poisson processes, the values of $A / \mathcal{S}$ in the simulations show scatter around this mean value. Figure 9 shows that this scatter is relatively narrow 
Table 9

Contents of the "Detections" Catalog Table

\begin{tabular}{|c|c|c|c|}
\hline Field & Units & Description & Has Errors? \\
\hline DetID & & A unique identifier for this detection & \\
\hline ObsID & & The unique 11-digit obsID of the dataset the detection occurred in. & \\
\hline Band & & $\begin{array}{l}\text { The band in which the detection occurred, } \\
(0=\text { total, } 1=\text { soft, } 2=\text { medium, } 3=\text { hard })\end{array}$ & \\
\hline DetFlag & & The detection flag as an integer value & \\
\hline $\operatorname{img\_ } x^{a}$ & pixels & The $x$-location of the detection in XRT SKY coordinates & \\
\hline $\operatorname{img\_ y} y^{a}$ & pixels & The $y$-location of the detection in XRT SKY coordinates & \\
\hline OffAxis & $\operatorname{arcmin}$ & The mean off-axis angle of the detection in this observation & \\
\hline RA & $\operatorname{deg}$ & R.A. (J2000) of the detection using the star tracker astrometry & Yes (statistical only) \\
\hline Dec & deg & Decl. (J2000) of the detection using the star tracker astrometry & Yes (statistical only) \\
\hline Err90 & $\operatorname{arcsec}$ & $90 \%$ conf. radial position error, statistical+systematic & \\
\hline RA_corr & $\operatorname{deg}$ & RA (J2000) of the detection using 2MASS/XRT astrometry & \\
\hline Dec_corr & $\operatorname{deg}$ & Declination (J2000) of the detection using 2MASS/XRT astrometry & \\
\hline Err90_corr & $\operatorname{arcsec}$ & $90 \%$ conf. radial position error using $2 \mathrm{MASS} / \mathrm{XRT}$ astrometry & \\
\hline$l$ & $\operatorname{deg}$ & Galactic longitude of the detection & \\
\hline$b$ & deg & Galactic latitude of the detection & \\
\hline Counts & & Number of events in the count-rate extraction region & \\
\hline BGCts & & The expected number of background events in the above region & \\
\hline FieldExposure & $\mathrm{s}$ & The on-axis exposure of the dataset the detection is in & \\
\hline $\mathrm{CF}$ & & The count-rate correction factor $(\kappa)$ & \\
\hline Rate & counts $\mathrm{s}^{-1}$ & The count rate of the detection & Yes \\
\hline ExposureFraction & & $\begin{array}{l}\text { The exposure time at the location of the } \\
\text { detection divided by the on-axis exposure }\end{array}$ & \\
\hline Cstat & & The $\mathcal{C}$ value from the PSF fit & \\
\hline Cstat_nosrc & & The $\mathcal{C}$ value calculated with normalization $=0$ & \\
\hline LogLikelihood & & The log-likelihood of the detection & \\
\hline $\mathrm{S} / \mathrm{N}$ & & The $\mathrm{S} / \mathrm{N}$ of the detection & \\
\hline Celldet_width & pixels & The size of the cell in which the detection was made & \\
\hline PSF_Radius & pixels & The radius of the circular region used in PSF-fitting & \\
\hline PSF & & Which PSF profile was selected by PSF-fitting & \\
\hline ol_warn & mag & $\begin{array}{l}\text { The number of magnitudes brighter than the } \\
\text { warning level of any cataloged star within } \\
30^{\prime \prime} \text { of the detection }\end{array}$ & \\
\hline FieldFlag & & The flag associated with the dataset the detection is in & \\
\hline NNDist & $\operatorname{arcsec}$ & The distance to the nearest other detection in this image & \\
\hline OKNNDist & $\operatorname{arcsec}$ & The distance to the nearest Good or Reasonable detection in this image & \\
\hline Num_snapshots & & How many snapshots are in the image containing the detection & \\
\hline Image BG & counts s ${ }^{-1}$ pixel $^{-1}$ & $\begin{array}{l}\text { The mean background level in the image, } \\
\text { according to the background map }\end{array}$ & \\
\hline MergeRadius & pixels & $\begin{array}{l}\text { The radius over which other detections in this image } \\
\text { are assumed to be aliases of this detection }\end{array}$ & \\
\hline SourceID & & $\begin{array}{l}\text { The identifier of the unique 1SXPS source this to } \\
\text { which this detection corresponds }\end{array}$ & \\
\hline
\end{tabular}

Note. ${ }^{\text {a }}$ The SKY coordinate system for an image depends on the position information used process the raw XRT data, thus may not be the same for user-processed data.

Table 10

Contents of the "Cross-correlations" Catalog Table

\begin{tabular}{lcl}
\hline \hline Field & Units & \multicolumn{1}{c}{ Description } \\
\hline 1SXPS_ID & & The name of the 1SXPS source \\
ExtCat_ID & & The name of the source in the external catalog \\
Catalog & The catalog containing the matched source \\
Distance & arcsec & The distance between the 1SXPS source and external catalog source \\
R.A. & deg & The R.A. (J2000) of the source in the external catalog \\
Decl. & deg & The Decl. (J2000) of the source in the external catalog \\
Err90 & arcsec & The 90\% confidence radial uncertainty in the external catalog position, including any systematic \\
\hline
\end{tabular}

(a Gaussian fit has $\sigma \sim 0.6$ ), and adding it to the count-rate uncertainty makes negligible difference to that value. Thus this effect can be safely neglected.

The bottom panel of Figure 9 shows the distribution of $(R-\mathcal{T}) / \sigma_{R}$. As can be seen, this distribution is significantly skewed with the catalog tending to overestimate the true count rate. This is simply the result of the Eddington bias (Eddington 1940): if the true source count rate is close to the detector limit then we detect those sources which Poisson noise makes appear brighter, but not those which are made fainter. 

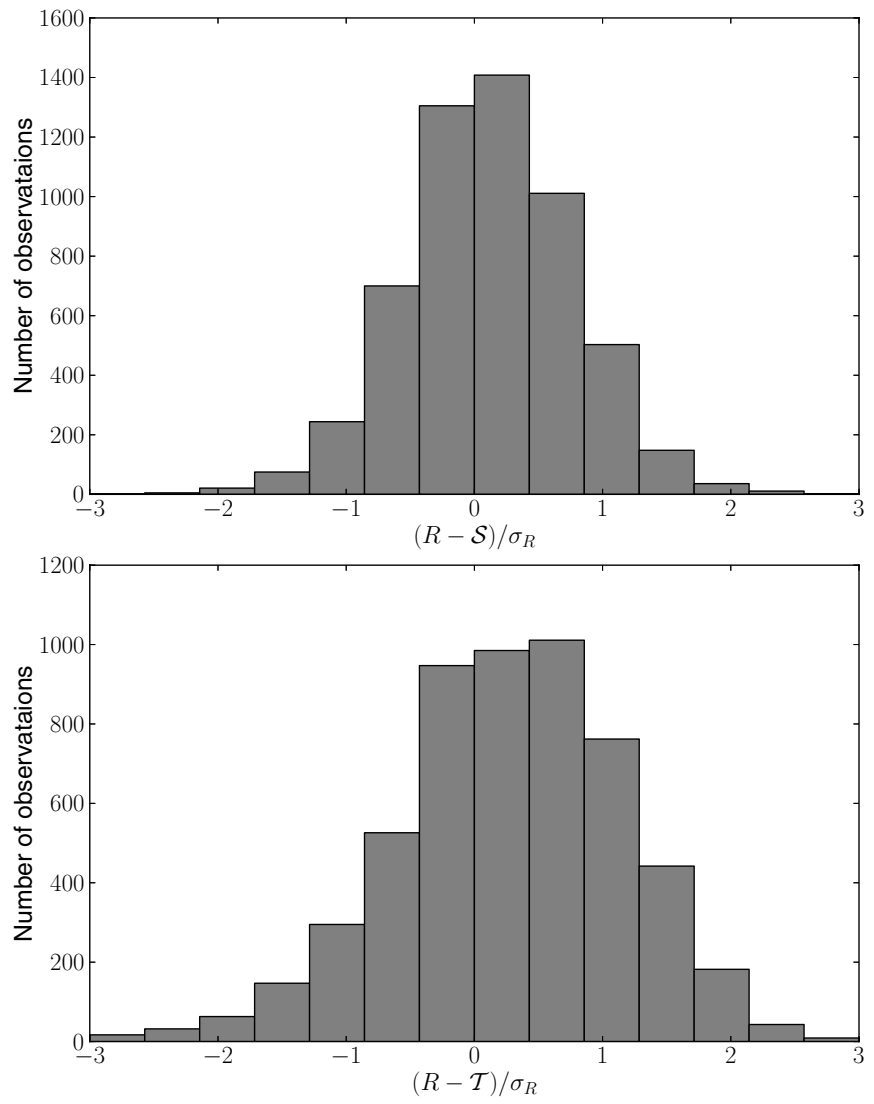

Figure 9. Histogram showing the accuracy of our count-rate reconstruction, based on simulation. Top: The difference between the measured and simulated source count rate, divided by the error on the measured value. Bottom: The difference between the measured and true source count rate, divided by the error on the measured value. The asymmetry in this plot is due to the Eddington bias.

\subsubsection{Eddington Bias}

To explore the magnitude of the Eddington Bias in our data, we simulated a further 20,000 images, again with the exposure time and background level drawn at random from the distributions seen in the catalog, and with the source fluxes drawn from a $\log N-\log S$ distribution. We then ran our catalog software on those images, recording both the "true" count rate from the simulation $(\mathcal{T})$ and the count rate $R$ determined by our software. In Figure 10 we show the distribution of the ratio $R / \mathcal{T}$ as a function of how many simulated events there were $\left(A_{c}\right)$ for the source in question. This shows that (unsurprisingly) the Eddington bias is very strong for the faintest sources in the catalog, with the count rates determined typically a factor of two too high. Although this bias lessens as we move to brighter sources, the distribution of rates recovered is still significantly asymmetric at $A_{c}=20$, however for sources with at least 30 events, the effect of the Eddington bias has all but disappeared.

\subsection{Variability Test}

We performed the Pearson's $\chi^{2}$ tests for variability on the sources in the 5,000 simulations created for Section 6.2. Since these sources are simulated with constant intensity (which is the null hypothesis of these tests) we expect that $10 \%$ of the sources will have a $P<0.1$ etc. Figure 11 shows that this is the result obtained. This does not provide information on how strong variability has to be before it is detected, however this is a function of variability type, exposure, source brightness, light curve sampling etc. and should be determined on a per-source basis.

\subsection{Spectroscopy}

The distribution of $\chi_{v}^{2}$ from the power-law and APEC model spectral fits shows a clustering around $\chi_{v}^{2}=1$ for both spectral models, as expected if those models are good representations of the data. About $25 \%$ of fits have $\chi_{v}^{2} \gg 1$, these represent cases where the simple spectral models we have used are not appropriate and more complex (e.g., multi-temperature) emission processes are likely involved. For those sources for which we have both a spectral fit with $\chi_{v}^{2}<1.5$ and an estimate of the spectral parameters derived from the hardness ratios, we show in Figure 12 a histogram of the HR-Fit/Fit, for both the observed flux and the emission parameter. This shows that the spectral parameters derived from the hardness ratios are reasonable.

\section{QUALITY FLAGS, FALSE POSITIVE RATE, AND CATALOG COMPLETENESS}

The quality flags described in Section 3.4 were calibrated such that the false positive rate in the catalog was $0.3 \%, 1 \%$ or $10 \%$ when Good, Good and Reasonable, or Good, Reasonable and Poor sources are included respectively. To calibrate these levels we again used simulations. Initially we performed a series of simulations of fixed exposure times $(1,2,5,10,20,40$ and $150 \mathrm{ks})$. We ran the catalog source detection software on each simulated image, and compared the list of detected sources with those simulated to determine the rate of false positives and therefore set the likelihood thresholds corresponding to each quality flag. The false positive rate proved to be a function of exposure time, and we defined the quality flags accordingly. To test these flag definitions over a range of exposures and background levels more representative of the catalog than the discrete exposures use above, we ran a further 20,000 simulations, drawing the exposure time and background level at random from the distribution of these values in the catalog datasets. We found it necessary to reclassify some sources as Bad based on their positional errors. We also found that at exposures shorter than $\sim 4 \mathrm{ks}$, the false positive rate never rose above $\sim 2 \%$, we therefore added a caveat that, for images shorter than $4 \mathrm{ks}$, the flag could only be Good or Reasonable. We ran a further 20,000 simulations to confirm that the results were stable. The formal definitions of the flags are given in Table 11; the false positive rate as a function of exposure time and quality flag is shown in Figure 13.

We used the results of the simulations above to measure the fraction of simulated sources detected as a function of $0.3-10 \mathrm{keV}$ source flux, exposure time and quality flag. Figure 14 shows the result. The median exposure time of the observations in the catalog is $1.5 \mathrm{ks}$, at which our procedure is $50 \%$ complete at $3 \times 10^{-13}$ erg cm$~^{-2} \mathrm{~s}^{-1}$, and $90 \%$ complete at $7 \times 10^{-13} \mathrm{erg} \mathrm{cm}^{-2} \mathrm{~s}^{-1}$. For the stacked images, the median exposure time is $6 \mathrm{ks}$, at which our catalog is $50 \%$ complete at $1 \times 10^{-13} \mathrm{erg} \mathrm{cm}^{-2} \mathrm{~s}^{-1}$, and $90 \%$ complete at $2 \times 10^{-13} \mathrm{erg} \mathrm{cm}^{-2} \mathrm{~s}^{-1}$.

\section{RESULTS AND DISCUSSION}

Our catalog contains 151,524 unique sources of which 98,762 are in our highest quality "clean" subsample (Good and 

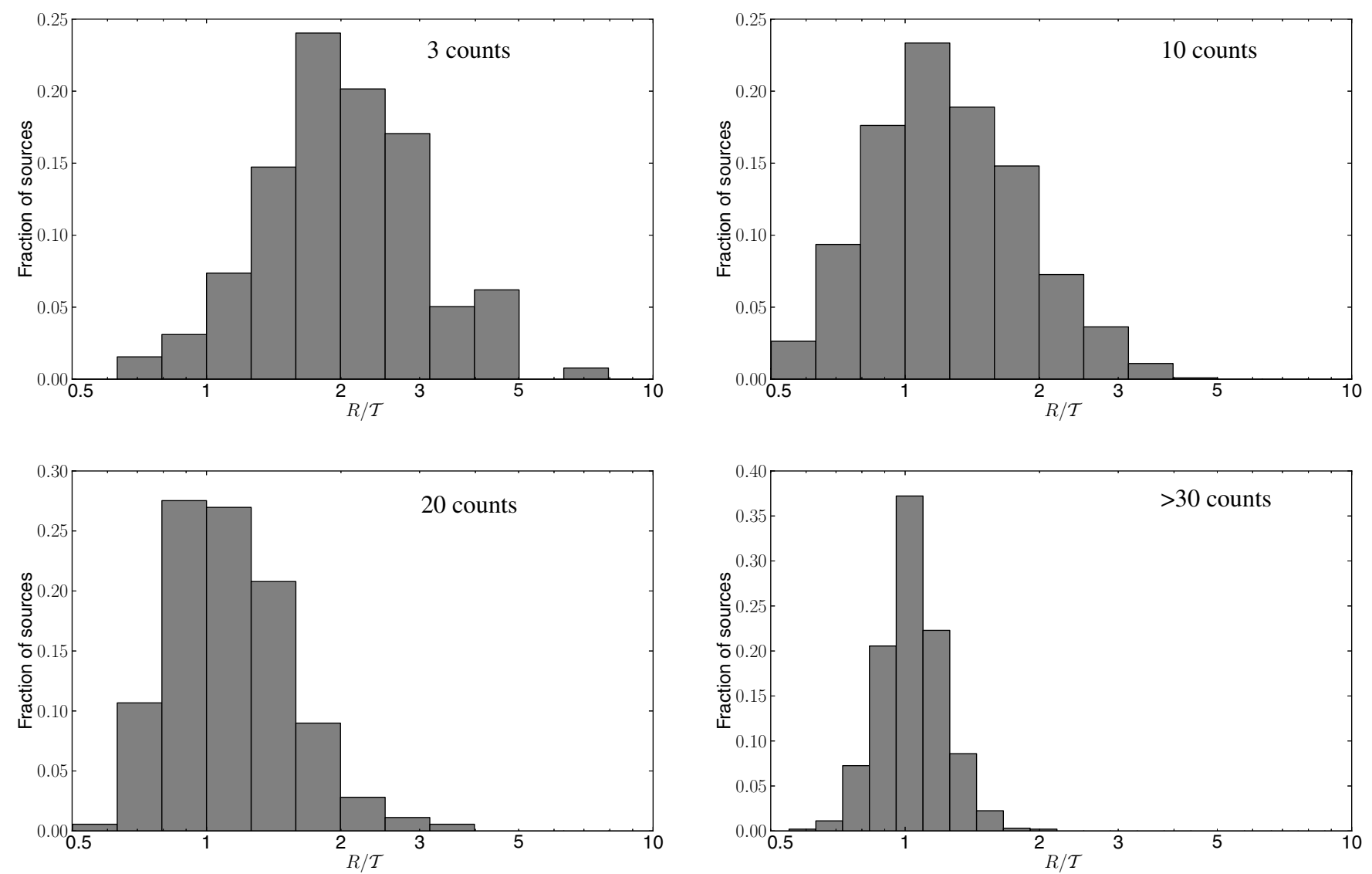

Figure 10. Effect of the Eddington Bias, showing the ratio of the measured count rate to the true count rate $(R / \mathcal{T})$ as a function of the number of simulated counts, $A_{c}$.

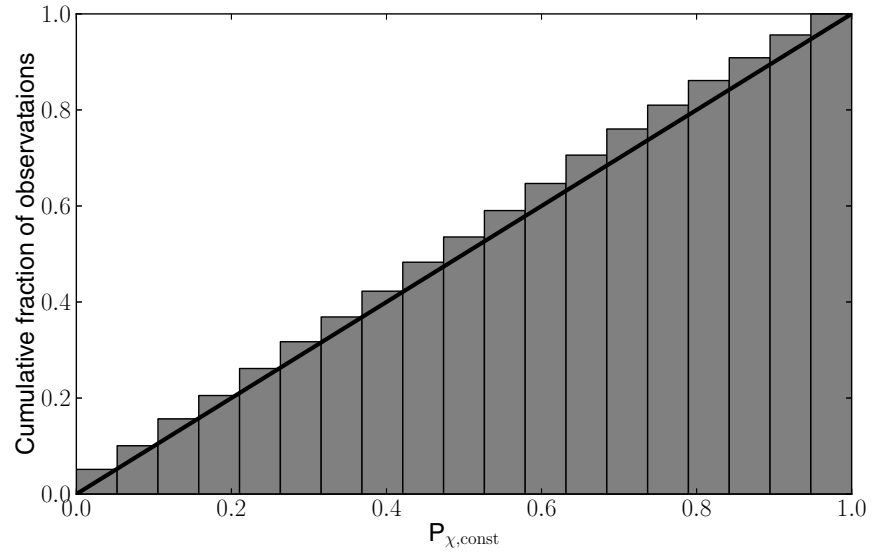

Figure 11. Cumulative probability distributions from the Pearson's $\chi^{2}$ variability test applied to the constant sources in 5000 simulated images. The black line shows the expected result which is well matched by the data.

Reasonable sources only, excluding those in fields containing diffuse emission). Table 12 shows the breakdown of the sources according to the detection and field flags. The distribution of fluxes in the clean and total samples is shown in Figure 15. Due to the effects of (in)completeness (Section 7) and the presence of the observation target object in our catalog, a $\log N-\log S$ calculation cannot be deduced directly from this figure-see Mateos et al. (2008) for a detailed discussion of the issues involved.

Due to the observing strategy of Swift, our catalog gives a unique insight into variability on multiple timescales. Excluding GRBs, 28,906 sources are found to be variable at the 3- $\sigma$ level
Table 11

Definitions of the Detection Flags

\begin{tabular}{ll}
\hline \hline Name & \multicolumn{1}{c}{ Definition } \\
\hline Good $(=0)$ & $L>18.52 E^{-0.051}$ \\
Reasonable $(=1)$ & $L \leqslant 18.52 E^{-0.051}(E<4 \mathrm{ks})$ \\
& $L>36.32 E^{-0.15}(4 \mathrm{ks}<E<40 \mathrm{ks})$ \\
& $L>9.73 E^{-0.024}(E \geqslant 40 \mathrm{ks})$ \\
& $L>86.55 E^{-0.29}(4 \mathrm{ks}<E<26 \mathrm{ks})$ \\
Poor $(=2)$ & $L>3.47 E^{0.027}(E \geqslant 26 \mathrm{ks})$ \\
& $L<L$ poor or any position err $( \pm$ R.A., decl. $)>25^{\prime \prime}$ \\
Bad $^{\text {a }}$ & As Good but in a region marked as containing artifacts. \\
\hline Value $=8$ & As Reasonable but in a region marked as containing \\
Value $=9$ & artifacts. \\
Value $=10$ & As Poor but in a region marked as containing artifacts. \\
Value $=16$ & As Good but in a region marked as containing diffuse \\
Value $=17$ & emission. \\
Value $=18$ & As Reasonable but in a region marked as diffuse \\
\hline
\end{tabular}

Notes. $L$ is the source likelihood value, and $E$ the exposure time in seconds.

${ }^{a} \mathrm{Bad}$ detections are not included in the catalog.

in at least one band or binning method. Figure 16 shows the distribution of the $\chi^{2}$ variability probability (Section 4.1) for the total-band light curves, GRBs have been excluded from this plot. A clear excess above the expected uniform distribution is seen at low probability of being constant, indicating a population of variable sources. Figure 17 shows an example light curve of one of these sources, 1SXPS J192427.2+240925, which appears to be short-lived transient that was only visible for three snapshots. This object was found by searching for sources 


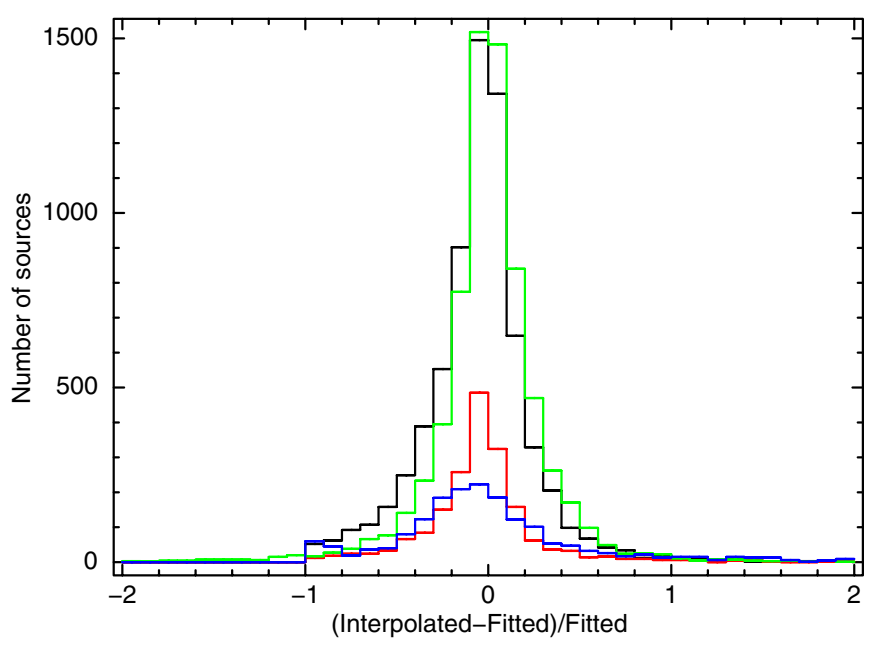

Figure 12. Difference between the spectral parameters derived from the hardness ratio and those from the spectral fit, divided by the spectrally fitted value. Only sources where the spectral fit had $\chi_{v}^{2}<1.5$ are shown. Black and red: observed flux from a power-law and APEC spectrum respectively. Green: photon index from a power-law spectrum. Blue: plasma temperature from an APEC spectrum.

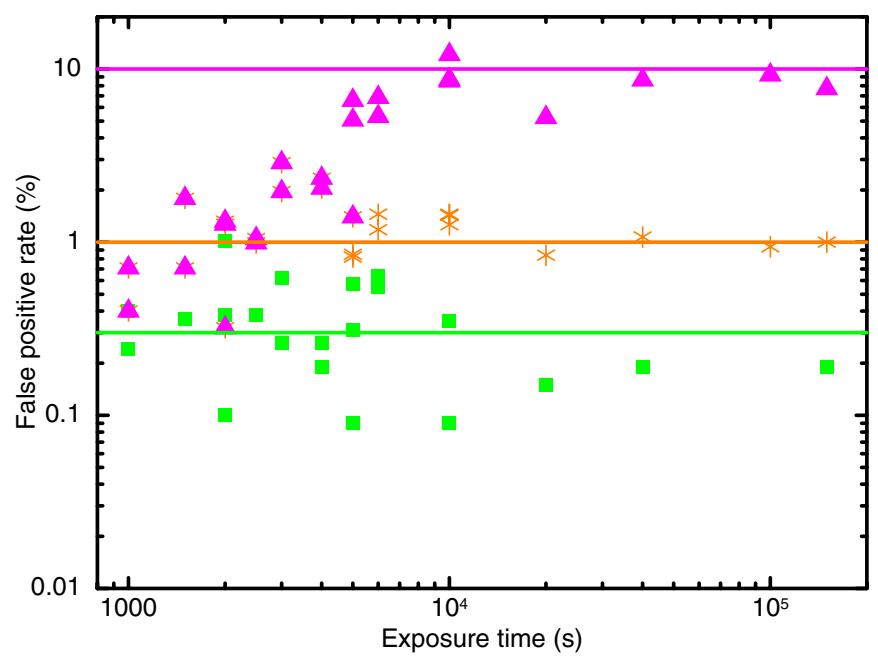

Figure 13. False positive rate measured from the various simulation runs, as a function of exposure time. Green: Good sources. Orange: Good and Reasonable sources. Magenta all sources. The horizontal lines represent the $0.3 \%, 1 \%$ and $10 \%$ levels.

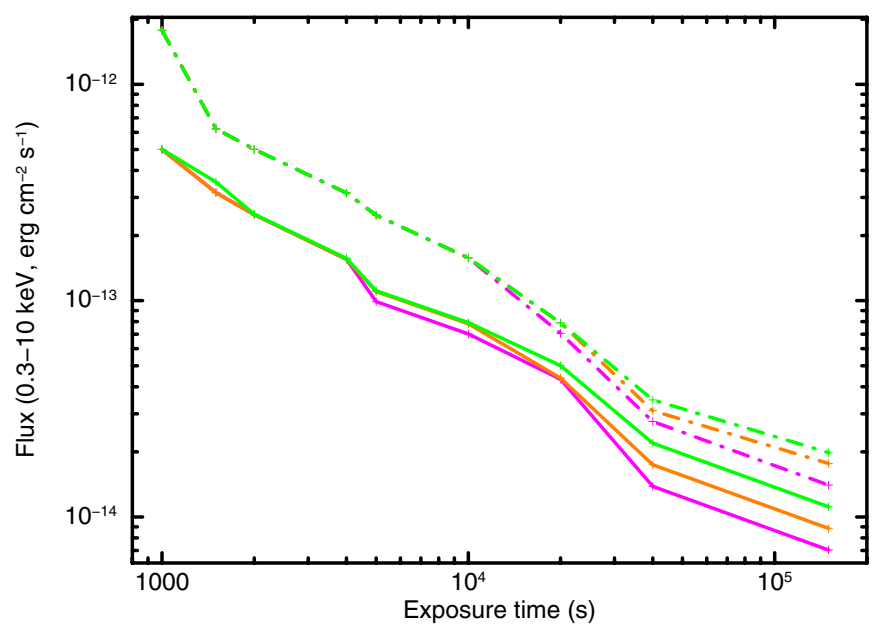

Figure 14. Completeness of our detection method as a function of exposure time and quality flag. The solid line is the $50 \%$ complete level, the dot-dashed line the $90 \%$ complete level. Green: Good sources. Orange: Good and Reasonable sources. Magenta all sources.
Table 12

The Number of 1SXPS Sources by Flag Values

\begin{tabular}{|c|c|}
\hline Flag Value & Num Sources \\
\hline \multicolumn{2}{|l|}{ Detection flags } \\
\hline \multicolumn{2}{|c|}{ In fields flagged as $\mathrm{OK}$} \\
\hline Good & $69,967(61 \%)$ \\
\hline Reasonable & $16,127(14 \%)$ \\
\hline Poor & $27,904(24 \%)$ \\
\hline \multicolumn{2}{|c|}{ In fields containing artifacts } \\
\hline Good & $9,856(42 \%)$ \\
\hline Reasonable & $2,812(12 \%)$ \\
\hline Poor & $5,557(23 \%)$ \\
\hline Other ${ }^{\mathrm{a}}$ & $5,433(23 \%)$ \\
\hline \multicolumn{2}{|c|}{ In fields containing diffuse emission } \\
\hline Good & $1,422(10 \%)$ \\
\hline Reasonable & $455(3 \%)$ \\
\hline Poor & $986(7 \%)$ \\
\hline Other $^{\mathrm{a}}$ & $11,005(79 \%)$ \\
\hline \multicolumn{2}{|c|}{ In all fields } \\
\hline Good & $81,245(54 \%)$ \\
\hline Reasonable & $19,394(13 \%)$ \\
\hline Poor & $34,447(23 \%)$ \\
\hline Other $^{\mathrm{a}}$ & $16,438(11 \%)$ \\
\hline \multicolumn{2}{|l|}{ Field flags } \\
\hline $\mathrm{OK}$ & $113,998(75 \%)$ \\
\hline Has artifacts & $23,658(16 \%)$ \\
\hline Has diffuse emission & $13,868(9 \%)$ \\
\hline
\end{tabular}

Note. " "Other" refers to sources that lie within a region marked by manual screening, i.e., sources with detection flags of eight or above. See Section 3.6.

in the clean catalog sample that had a low probability of being constant and no counterpart found in the external catalog crosscorrelation (apart from a USNO-B1 or 2MASS object). Further investigation revealed a single $K=16.06 \mathrm{mag}$ stellar object in the UKIDSS Galactic Plane Survey in the XRT error region. This object is not in the USNO-B1 catalog, which has a limiting sensitivity of $V \sim 21 \mathrm{mag}$. It thus seems likely that this object is an $\mathrm{M}$ dwarf star within $1 \mathrm{kpc}$, and that the XRT detected a coronal flare from it which lasted a few hours.

In Figure 7 we showed the area of (HR1, HR2) space permitted by simple spectral models (a single absorber and emission component). Figure 18 shows the distribution of 1SXPS sources in this space, revealing a significant number which do not lie within the range permitted by these simple models. Indeed $\sim 14,300(9 \%)$ of all sources in the catalog are not consistent with the single-component power-law or APEC models, at the $3 \sigma$ level. Figure 18 also shows the distributions of the individual hardness ratios.

\subsection{Comparison with Other Catalogs}

The combination of sensitivity and sky coverage of this cata$\log$ means it occupies the area of parameter space between the deep-and-narrow surveys such as 3XMM-DR4, 1CSC (Evans et al. 2010) and the Chandra BMW catalog (Romano et al. 2008); and the shallow and wide surveys such as the Rosat All-Sky Survey (Voges et al. 1999) and the XMM Slew Survey (Saxton et al. 2008). The number of sources in the 1SXPS cata$\log$ with no counterpart in the set of catalogs shown in Table $6^{22}$

\footnotetext{
22 Excluding the 2MASS and USNO-B1 catalogs as the high spatial density of sources in these catalogs makes it hard to be certain of association with the 1SXPS source.
} 


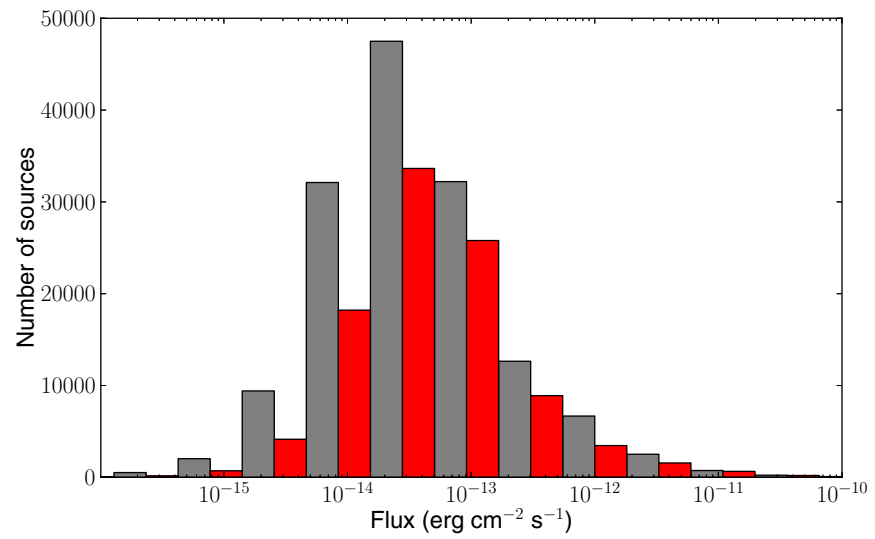

Figure 15. Distribution of the $0.3-10 \mathrm{keV}$ mean observed flux (derived assuming a power-law spectrum) for the sources in our catalog. The gray bins are for all sources, the red bins (darker gray in the printed journal) are for those in the "clean" subsample. The flux shown is taken from the spectral fit, where available; otherwise it comes from the hardness ratio interpolation, and if this is not available then from the fixed spectrum (see Section 4.2 for details).

(A color version of this figure is available in the online journal.)

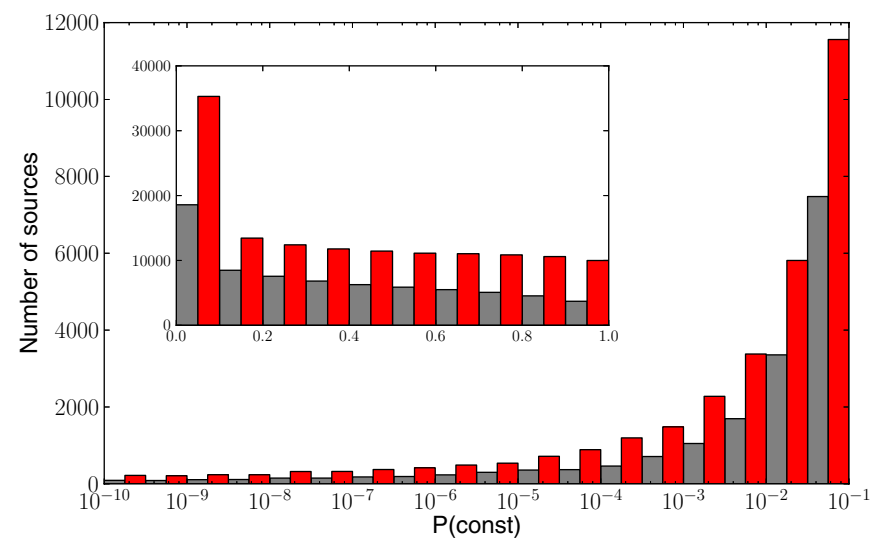

Figure 16. Distribution of the $\chi^{2}$-derived probability that a source is constant for the 1SXPS catalog sources, excluding GRB afterglows. The gray data are for inter-snapshot variability, the red bins (darker gray in the printed journal) for inter-observation. The inset shows the entire probability range, over which a population of constant sources would show equal numbers of objects in each bin: the sharp spike at $P<0.1$ indicates a population of variable sources; the main plot shows a magnified view (with a logarithmic probability axis) of this region.

(A color version of this figure is available in the online journal.)

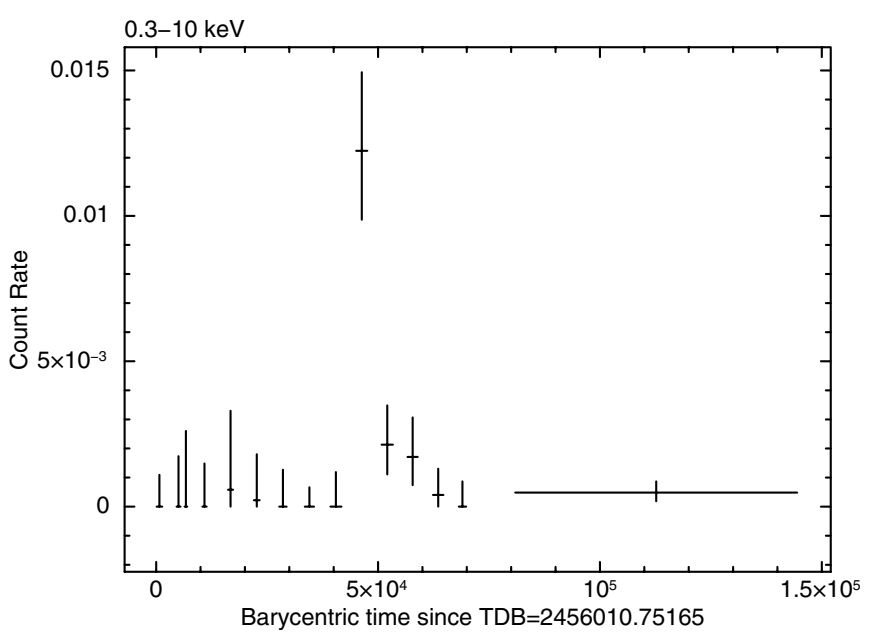

Figure 17. Total-band light curve of 1SXPS J192427.2+240925 with one bin per snapshot. This is a short-lived transient, newly discovered in the 1SXPS catalog.
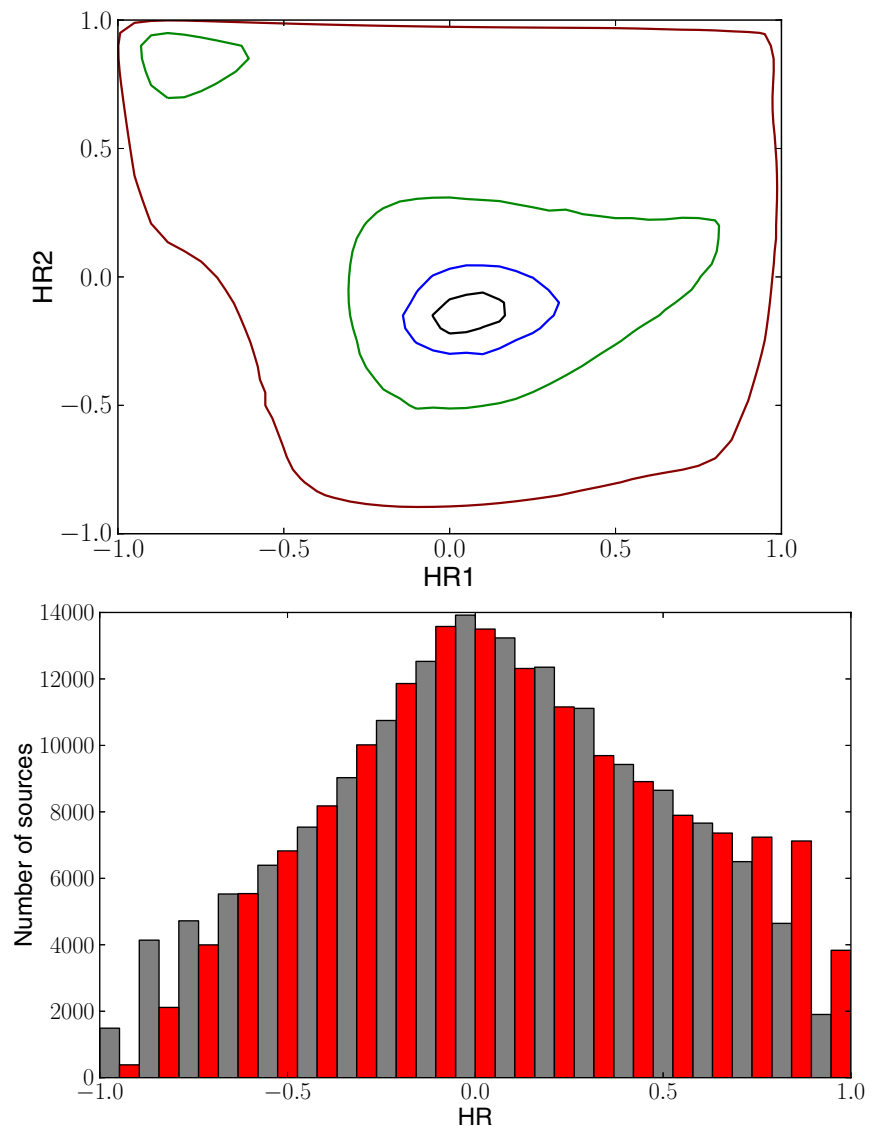

Figure 18. Top: contour plot showing the (HR1, HR2) space occupied by the 1SXPS sources, after smoothing over the error range of the individual sources. The contours show the areas $25 \%, 50 \%, 75 \%$, and $90 \%$ of the peak density. Bottom: the distribution of the individual HR values. Gray: HR1, Red: HR2 (darker gray in the printed journal).

(A color version of this figure is available in the online journal.)

is $68,638(45 \%)$ sources from the full catalog, and 33,282 (34\%) sources in the clean sample. In part this is simply due to the limited overlap between surveys: $31 \%$ of our fields have a $3 \mathrm{XMM}$ source within the field of view (including those undetected in our catalog), and $14 \%$ have an SDSS quasar in the field, giving an idea of the size of the overlap.

The fields of the earlier XRT catalogs of Puccetti et al. (2011) and D'Elia et al. (2013) are included in the sample we have used. We found many sources not in those catalogs, partly because we included more data, but mainly because of the difference in strategy between the catalogs. Puccetti et al. (2011) focused on only stacked images of GRB fields (totaling 374 fields compared to our 7343); D'Elia et al. (2013) used a much larger sample than Puccetti et al. (2011), similar in size to ours (35,011 observations compared to the 48,932 in our catalog 23 ), however, they used a higher $\mathrm{S} / \mathrm{N}$ threshold than we did, and did not combine images thus limiting the sensitivity achieved. Our approach combines the advantages of both of these methods. Further, our improved detection system is significantly more sensitive than the XIMAGEbased approach employed in the earlier catalogs: for example simulations showed that in a $2 \mathrm{ks}$ image for a source with a count rate of 0.004 counts $\mathrm{s}^{-1}\left(\sim 2 \times 10^{-13} \mathrm{erg} \mathrm{cm}^{-2} \mathrm{~s}^{-1}, 0.3-10 \mathrm{keV}\right)$ our system is $37 \%$ complete, which is 1.5 times as complete as

\footnotetext{
23 The difference arising partly because our sample extends ten months after the D'Elia et al. (2013) sample, and partly because we set a lower limit of $100 \mathrm{~s}$ of PC mode data, where they use $500 \mathrm{~s}$.
} 
$30^{\circ}$

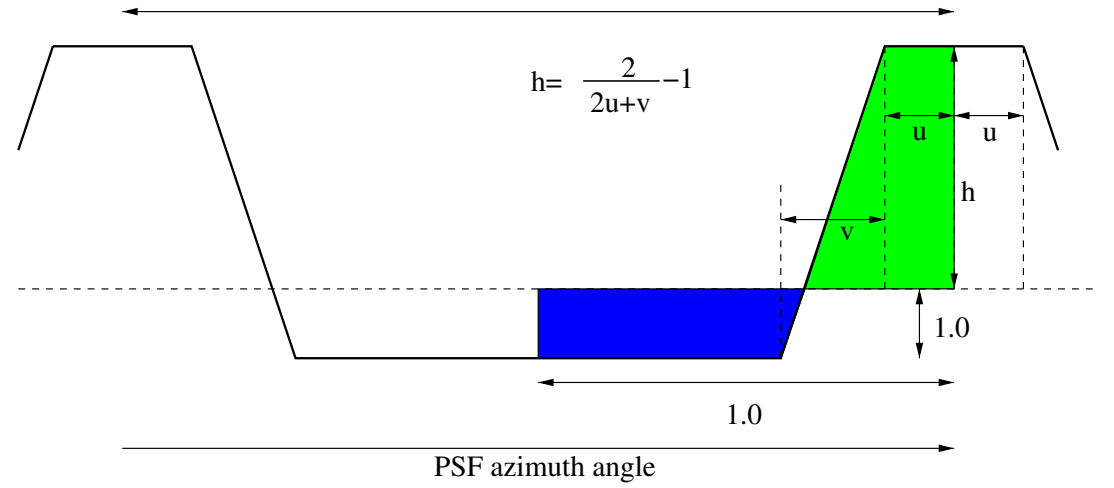

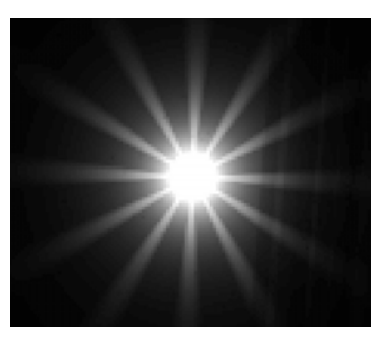

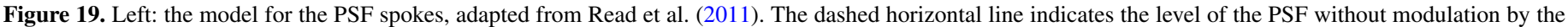

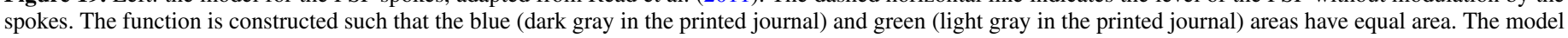

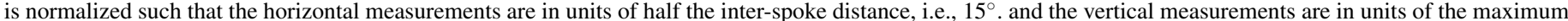

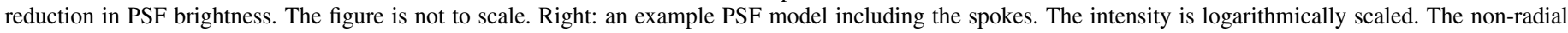
structure is caused by the exposure map.

(A color version of this figure is available in the online journal.)

the XIMAGE system; the same is true for a source of count rate 0.002 counts $\mathrm{s}^{-1}\left(\sim 8 \times 10^{-14} \mathrm{erg} \mathrm{cm}^{-2} \mathrm{~s}^{-1}, 0.3-10 \mathrm{keV}\right)$ in a $5 \mathrm{ks}$ image; the false positive rates in the two approaches were found to be similar. This combination of factors explains the number of sources present in our catalog that were not found in the earlier XRT catalogs.

The X-ray sky is highly variable, as evidenced by Figure 16, and to some extent all catalogs are biased in their contents toward sources in high states. For example Starling et al. (2011) used Swift to observe 94 unidentified X-ray sources from the XMM Slew Survey with much greater sensitivity than that survey but only detected $30 \%$ of the $X M M$ objects. Nonetheless, this catalog, with its census of variability and useful combination of moderate exposure and moderate sensitivity, will serve as a useful baseline for future missions such as eRosita and provides valuable information on the nature of variable sources which will be part of the unresolved background for missions like LOFT.

P.A.E., J.P.O., A.P.B., K.L.P., C.P. and C.J.M. acknowledge support from the UK Space Agency. D.N.B. and J.A.K. acknowledge support from NASA contract NAS5-00136. G.T. acknowledges support from ASI-INAF grant I/004/11/0. We thank Simon Vaughan for helpful discussions during the preparation of this paper and Paul O'Brien for feedback on the manuscript. We also thank the anonymous referee and the Scientific Editor for insightful and helpful comments. This work made use of data supplied by the UK Swift Science Data Center at the University of Leicester. This research has made use of the XRT Data Analysis Software (XRTDAS) developed under the responsibility of the ASI Science Data Center (ASDC), Italy. This research has made use of the SIMBAD database, operated at CDS, Strasbourg, France.

\section{APPENDIX}

\section{MODIFICATIONS TO THE PSF PROFILE}

The standard PSF of the Swift-XRT was calibrated by Moretti et al. (2007) and is modelled as a radially symmetric King function:

$$
P(R) \propto\left[1+\left(\frac{R}{R_{C}}\right)^{2}\right]^{-\beta}
$$

The real PSF shows deviations from this profile due to the presence of "spokes" caused by the shadowing of light by the mirror support structure. Read et al. (2011) performed a comprehensive analysis of this effect for $X M M$ and found that modulating the azimuthal variation of the PSF by a trapezoidal function, shown in Figure 19, gave a good representation of the PSF spokes. We applied this model to the XRT, first modifying it to account for the smaller number of spokes in XRT data (12, compared to 16 for $X M M$ ); and then determined the function parameters by fitting the model to an XRT dataset. If the model depicted in Figure 19 is $f(\theta)$, then the PSF is given by

$$
P(R, \theta)=P(R)[1+N(R) f(\theta)],
$$

where $N(R)$ reflects the fact that the strength of the spoking effect is a function of radius within the PSF. This is a simple function with four parameters: $N_{\mathrm{pk}}, R_{\mathrm{min}}, R_{\mathrm{pk}}$ and $R_{\mathrm{max}} . N(R)$ is given thus:

$$
\begin{aligned}
N(R)= & 0 \\
& \left(R<R_{\mathrm{min}} \text { or } R>R_{\mathrm{max}}\right) \\
N(R)= & \left(\frac{N_{\mathrm{pk}}}{R_{\mathrm{pk}}-R_{\min }}\right)\left(R-R_{\mathrm{min}}\right) \\
& \left(R_{\mathrm{min}}<R<R_{\mathrm{pk}}\right) \\
N(R)= & N_{\mathrm{pk}}-\left(\frac{N_{\mathrm{pk}}}{R_{\mathrm{max}}-R_{\mathrm{pk}}}\right)\left(R-R_{\mathrm{pk}}\right) \\
& \left(R_{\mathrm{pk}} \leqslant R \leqslant R_{\mathrm{max}}\right) .
\end{aligned}
$$

At $R<R_{\min }$ or $R>R_{\max } N(R)=0$, at $R_{\min }<R<R_{\mathrm{pk}}$ $N(R)$ increases linearly to $N_{\mathrm{pk}}$ and then it decreases linearly again to 0 at $R_{\max }$.

A non-piled-up point source would ideally be used to fit the PSF spoke parameters however this proved impossible. Each snapshot of Swift data has a slightly different pointing position and roll angle so to model the PSF spokes we had to use only a single snapshot of data. Pile up becomes an issue at around 0.6 count $\mathrm{s}^{-1}$ so single-snapshot images of non piled-up sources did not contain enough counts for us to perform a reliable fit to the relatively weak PSF spoke effect. We therefore used a brighter but piled up source, accepting that this will give us a model to 
Table 13

The Parameters for the PSF Spoke Model Used in Our Background Mapping Tool

\begin{tabular}{lc}
\hline \hline Parameter & Value \\
\hline$u$ & $0.0574^{\mathrm{a}}$ \\
$v$ & $0.1512^{\mathrm{a}}$ \\
$R_{\min }$ & $5^{\prime \prime} 6$ \\
$R_{\mathrm{pk}}$ & $42^{\prime \prime} 4$ \\
$R_{\max }$ & $238^{\prime \prime}$ \\
$N_{\mathrm{pk}}$ & 0.21 \\
\hline
\end{tabular}

Note. ${ }^{\mathrm{a}} u$ and $v$ are in units of half a phase, i.e., $15^{\circ}$.

the PSF spokes which is probably imperfect for non-piled-up source, but better than no model at all. The parameters of this fit are given in Table 13.

As well as adding in the PSF spokes it was sometimes necessary to incorporate OOT events into the background map when modeling sources. Out-of-time events are events detected while the CCD is being read out, spreading the $y$-position of those events along the entire column. Since the deadtime in PC mode is equal to 0.004 times the exposure time, the count rate of OOT events in a given CCD column is simply 0.004 times the number of in-time events in that column. We estimate the latter value by reading the number of events in a 41 pixel high region centered on the source and then multiply this by 0.004 and divide it by 600 (the number of rows on the CCD). We then add the resultant value to the background map for every pixel on that row. We perform this for an 11 pixel wide region centered on the source. This is only done for sources brighter than 3 counts $\mathrm{s}^{-1}$ since below this level OOT events are insignificant compared to the background.

\section{REFERENCES}

Abbey, T., Carpenter, J., Read, A., et al. 2006, in ESA Special Publication, Vol. 604, The X-ray Universe 2005, ed. A. Wilson (Noordwijk: ESA), 943

Burrows, D. N., Hill, J. E., Nousek, J. A., et al. 2005, SSRv, 120, 165
Butler, N. R. 2007, AJ, 133, 1027

Calabretta, M. R., \& Greisen, E. W. 2002, A\&A, 395, 1077

Cash, W. 1979, ApJ, 228, 939

Churazov, E., Gilfanov, M., Forman, W., \& Jones, C. 1996, ApJ, 471, 673

D’Elia, V., Perri, M., Puccetti, S., et al. 2013, A\&A, 551, A142

Eddington, A. S. 1940, MNRAS, 100, 354

Evans, I. N., Primini, F. A., Glotfelty, K. J., et al. 2010, ApJS, 189, 37

Evans, P. A., Beardmore, A. P., Page, K. L., et al. 2009, MNRAS, 397, 1177

Gehrels, N. 1986, ApJ, 303, 336

Gehrels, N., Chincarini, G., Giommi, P., et al. 2004, ApJ, 611, 1005

Gioia, I. M., Maccacaro, T., Schild, R. E., et al. 1990, ApJS, 72, 567

Goad, M. R., Tyler, L. G., Beardmore, A. P., et al. 2007, A\&A, 476, 1401

Greisen, E. W., \& Calabretta, M. R. 2002, A\&A, 395, 1061

Høg, E., Fabricius, C., Makarov, V. V., et al. 2000, A\&A, 363, 385

Kennea, J. A., Burrows, D. N., Wells, A., et al. 2005, Proc. SPIE, 5898, 341

Kraft, R. P., Burrows, D. N., \& Nousek, J. A. 1991, ApJ, 374, 344

Mateos, S., Warwick, R. S., Carrera, F. J., et al. 2008, A\&A, 492, 51

Monet, D. G., Levine, S. E., Canzian, B., et al. 2003, AJ, 125, 984

Moretti, A., Pagani, C., Cusumano, G., et al. 2009, A\&A, 493, 501

Moretti, A., Perri, M., Capalbi, M., et al. 2007, Proc. SPIE, 6688, 14

Park, T., Kashyap, V. L., Siemiginowska, A., et al. 2006, ApJ, 652, 610

Pearson, K. 1900, PMag, 50, 157

Protassov, R., van Dyk, D. A., Connors, A., Kashyap, V. L., \& Siemiginowska, A. 2002, ApJ, 571, 545

Puccetti, S., Capalbi, M., Giommi, P., et al. 2011, A\&A, 528, A122

Read, A. M., Rosen, S. R., Saxton, R. D., \& Ramirez, J. 2011, A\&A, 534, A34

Romano, P., Campana, S., Mignani, R. P., et al. 2008, A\&A, 488, 1221

Samus, N. N., Kazarovets, E. V., Kireeva, N. N., Pastukhova, E. N., \& Durlevich, O. V. 2010, OAP, 23, 102

Saxton, R. D., Read, A. M., Esquej, P., et al. 2008, A\&A, 480, 611

Schneider, D. P., Hall, P. B., Richards, G. T., et al. 2007, AJ, 134, 102

Skrutskie, M. F., Cutri, R. M., Stiening, R., et al. 2006, AJ, 131, 1163

Smith, R. K., Brickhouse, N. S., Liedahl, D. A., \& Raymond, J. C. 2001, ApJL, 556, L91

Starling, R. L. C., Evans, P. A., Read, A. M., et al. 2011, MNRAS, 412,1853

Tundo, E., Moretti, A., Tozzi, P., et al. 2012, A\&A, 547, A57

Ueda, Y., Ishisaki, Y., Takahashi, T., Makishima, K., \& Ohashi, T. 2005, ApJS, 161,185

Voges, W., Aschenbach, B., Boller, T., et al. 1999, A\&A, 349, 389

Wald, A., \& Wolfowitz, J. 1940, Annals Math. Stat., 11, 147

Warren, W. H., Jr., \& Hoffleit, D. 1987, BAAS, 19, 733

Watson, M. G., Schröder, A. C., Fyfe, D., et al. 2009, A\&A, 493, 339

Willingale, R., Starling, R. L. C., Beardmore, A. P., Tanvir, N. R., \& O'Brien, P. T. 2013, MNRAS, 431, 394

Wilms, J., Allen, A., \& McCray, R. 2000, ApJ, 542, 914 